\title{
Repetitive Diffuse Mild Traumatic Brain Injury Causes an Atypical Astrocyte Response and Spontaneous Recurrent Seizures
}

\author{
Coleksii Shandra, ${ }^{1 *}$ Alexander R. Winemiller, ${ }^{1 \star}$ Benjamin P. Heithoff,,${ }^{1,2}$ Carmen Munoz-Ballester, ${ }^{1}$ \\ ๑Kijana K. George, ${ }^{1,4} \oplus^{-}$Michael J. Benko, ${ }^{1,5}$ Ivan A. Zuidhoek, ${ }^{1,4}$ Michelle N. Besser, ${ }^{1,3}$ @Dallece E. Curley, ${ }^{1,3}$ \\ (DG. Franklin Edwards III, ${ }^{1,3,4}$ Anroux Mey, ${ }^{1,3}$ Alexys N. Harrington, ${ }^{1}$ Jeremy P. Kitchen, ${ }^{1,4}$ and $@$ Stefanie Robel ${ }^{1,2,3}$ \\ ${ }^{1}$ Fralin Biomedical Research Institute at Virginia Tech Carilion, Roanoke 24016, Virginia, ${ }^{2}$ Department of Biological Sciences, ${ }^{3}$ School of Neuroscience, \\ Virginia Tech, Blacksburg 24060, Virginia, ${ }^{4}$ Graduate Program in Translational Biology, Medicine, and Health, Virginia Tech, Roanoke 24016, Virginia, and \\ ${ }^{5}$ VTCSOM-Carilion Clinic Department of Neurosurgery, Blacksburg 24014, Virginia
}

Focal traumatic brain injury (TBI) induces astrogliosis, a process essential to protecting uninjured brain areas from secondary damage. However, astrogliosis can cause loss of astrocyte homeostatic functions and possibly contributes to comorbidities such as posttraumatic epilepsy (PTE). Scar-forming astrocytes seal focal injuries off from healthy brain tissue. It is these glial scars that are associated with epilepsy originating in the cerebral cortex and hippocampus. However, the vast majority of human TBIs also present with diffuse brain injury caused by acceleration-deceleration forces leading to tissue shearing. The resulting diffuse tissue damage may be intrinsically different from focal lesions that would trigger glial scar formation. Here, we used mice of both sexes in a model of repetitive mild/ concussive closed-head TBI, which only induced diffuse injury, to test the hypothesis that astrocytes respond uniquely to diffuse TBI and that diffuse TBI is sufficient to cause PTE. Astrocytes did not form scars and classic astrogliosis characterized by upregulation of glial fibrillary acidic protein was limited. Surprisingly, an unrelated population of atypical reactive astrocytes was characterized by the lack of glial fibrillary acidic protein expression, rapid and sustained downregulation of homeostatic proteins and impaired astrocyte coupling. After a latency period, a subset of mice developed spontaneous recurrent seizures reminiscent of PTE in human TBI patients. Seizing mice had larger areas of atypical astrocytes compared with nonseizing mice, suggesting that these atypical astrocytes might contribute to epileptogenesis after diffuse TBI.

Key words: astrogliosis; concussion; diffuse TBI; gap junctions; neuroinflammation; posttraumatic epilepsy

\section{Significance Statement}

Traumatic brain injury (TBI) is a leading cause of acquired epilepsies. Reactive astrocytes have long been associated with seizures and epilepsy in patients, particularly after focal/lesional brain injury. However, most TBIs also include nonfocal, diffuse injuries. Here, we showed that repetitive diffuse TBI is sufficient for the development of spontaneous recurrent seizures in a subset of mice. We identified an atypical response of astrocytes induced by diffuse TBI characterized by the rapid loss of homeostatic proteins and lack of astrocyte coupling while reactive astrocyte markers or glial scar formation was absent. Areas with atypical astrocytes were larger in animals that later developed seizures suggesting that this response may be one root cause of epileptogenesis after diffuse TBI.

\section{Introduction}

Astrocytes carry out many homeostatic tasks crucial for normal brain function. They are in close spatial relationship to synapses ensuring low extracellular neurotransmitter and potassium concentrations essential for neuronal health and optimal function. CNS injury causes changes in astrocyte protein and gene expres- 
sion patterns and morphology (Sofroniew, 2009; Zamanian et al., 2012; Götz et al., 2015; Levine et al., 2016; Anderson et al., 2016). Astrogliosis is also crucial to protecting uninjured brain areas from secondary damage (Sofroniew, 2015). A site of primary focal brain damage becomes surrounded by layers of reactive astrocytes (Robel, 2017), which form a protective scar that seals off injured areas. Removal of these scar-forming astrocytes results in larger lesion sizes and worsened functional outcomes (Bush et al., 1999; Faulkner et al., 2004; Anderson et al., 2016).

However, this astrogliosis process can precede, and is essential to, the progression of many of neurological diseases including epilepsy (Ortinski et al., 2010; Lioy et al., 2011; Verkhratsky et al., 2012; Tong et al., 2014; Pekny et al., 2016). Evidence from human subjects and animal models demonstrates that reactive astrocytes lose their homeostatic functions thereby contributing to epileptogenesis (Wetherington et al., 2008; Robel and Sontheimer, 2016). Astrogliosis is present in lesional symptomatic epilepsies (Pollen and Trachtenberg, 1970; Niquet et al., 1994; Foresti et al., 2011; Steinhäuser et al., 2012; Bedner et al., 2015) and surgical removal of the glial scar alongside epileptic foci can provide seizure control in patients with temporal lobe epilepsy (Marks et al., 1995; Kumar et al., 2003).

TBI affects $>10$ million people annually and is induced by a mechanical force to the head. Approximately $2.9-50 \%$ of these TBI patients are at risk for developing epilepsy (Christensen et al., 2009; Lowenstein, 2009; Ferguson et al., 2010; Abou-Abbass et al., 2016), sometimes even years after the original insult. The risk for developing epilepsy is highest after focal-penetrating TBI, for example, caused by a gunshot or stab wound (Frey, 2003). Such focal injuries are associated with glial scar formation, but affect $<10 \%$ of all TBI patients. More frequently, TBIs combine focal and diffuse brain damage. In diffuse brain injury, accelerationdeceleration-rotational forces shear the brain tissue as a result of differing momentum between adjacent connected tissue masses of different densities. Diffuse TBI has been reported to increase the incidence of posttraumatic epilepsy (PTE) (Annegers et al., 1980, 1998; Ferguson et al., 2010). However, diffuse TBI occurs in isolation only in mild/concussive TBIs because this type by definition does not present with focal injury detectable by computed tomography (CT) imaging (Management of Concussion/mTBI Working Group, 2009). However, there is controversy in the field about whether mild TBI/concussion, which represents $75-86 \%$ of all TBIs (National Center for Injury Prevention and ControlCenters for Disease Control and Prevention, 2003), is sufficient for the development of PTE. Some studies did not find an increase in epilepsy incidence in postconcussive patients (Frey, 2003; Wennberg et al., 2018), whereas others did find an increased risk (Christensen et al., 2009; Ferguson et al., 2010; Christensen, 2012). Further confusion arises when epilepsy incidence rates after mild TBI $(2.9 \%$ or $4.4 \%)$ are compared with those in the general population (1.5\%) (Ferguson et al., 2010; Christensen, 2012), which both appear low overall. Unfortunately, the cause of epilepsy in the general population is often unknown. Awareness of risks associated with mild TBI/concussion and repetition of those injuries has only become commonplace in the last few years and one in nine people do not seek medical help (Centers for Disease Control). Therefore, medical records might lack

${ }^{*} 0 . S$. and A.R.W. contributed equally to this work.

Correspondence should be addressed to Stefanie Robel at srobel@vt.edu.

https://doi.org/10.1523/JNEUROSCI.1067-18.2018

Copyright $\odot 2019$ the authors $\quad 0270-6474 / 19 / 391945-20 \$ 15.00 / 0$ complete information about a history of mild TBI, making it impossible to link these injuries with seizures.

Ultimately, animal models that isolate diffuse TBI and do not present with focal lesions are needed to confirm the presence or lack of spontaneous recurrent seizures after mild/concussive TBI. Such a model of PTE is also necessary to identify cellular and molecular mechanisms contributing to epileptogenesis after diffuse TBI regardless of whether it is caused by mild or more severe forms of TBI.

\section{Materials and Methods}

\section{Animals}

Twelve- to 16-week-old C57BL/6 or Aldh1l1-eGFP//FVB/N mice of both sexes were used and animal sex is specified for each experiment in Figures 1-1 (available as Extended Data), 2-1 (available as Extended Data), 3-1 (available as Extended Data), 4-1 (available as Extended Data), 5-1 (available as Extended Data), 6-1 (available as Extended Data), 7-1 (available as Extended Data), and 8-1 (available as Extended Data). Many TBI and epilepsy studies have been performed in C57BL/6 mice. To ensure consistency and to allow for comparison of our data with other studies, we chose this strain for experiments that did not require reporter labeling of astrocytes. For some imaging experiments, we used the Aldh1l1-eGFP mouse line, which was backcrossed to $\mathrm{FVB} / \mathrm{N}$ for $>10$ generations (transgene expression can be modulated by the genetic background and FVB/N appears to be more permissive of transgene expression). Therefore, a pilot study assessed differences in the effect of diffuse TBI on FVB/N and C57BL/6 mice (see Fig. 1). Mice were bred in-house; some C57BL/6 mice were purchased from Harlan/Envigo Laboratories or The Jackson Laboratory. All animal procedures were approved and performed according to the guidelines of the Institutional Animal Care and Use Committee of Virginia Polytechnic Institute and State University (Virginia Tech) and were conducted in compliance with the National Institutes of Health's Guide for the Care and Use of Laboratory Animals.

Mouse strains used were as follows: C57BL/6 (JAX \#000664), FVB/N (JAX \#001800), Aldh1l1-eGFP (RRID:MMRRC_011015-UCD) backcrossed to FVB/N for $>10$ generations, GCamp5G (JAX \#024477)/ mGFAP-Cre 77.6 (kindly provided by Michael Sofroniew, UCLA) both C57BL/6; and Aldh1l1-tdTomato (RRID:MMRRC_036700-UCD; mixed FVB/N//Crl:CD1(ICR) backcrossed to Crl:CD1(ICR) for three generations.

\section{Impact acceleration (weight drop) injury}

Mice were injured using an impact acceleration model based on the modified Marmarou model (Foda and Marmarou, 1994; Marmarou et al., 1994, 2009) that induces diffuse TBI. Severity of the injury can be adjusted by modifying the drop height, the mass of the weight, the properties of the foam pad, and the interinjury interval. We calibrated this model to induce a repetitive mild/concussive injury (Nichols et al., 2016) in adult mice 12-16 weeks of age by adjusting drop height and interinjury interval (see Fig. 1). The mouse was anesthetized with $3 \%$ isoflurane gas in an induction chamber for $5 \mathrm{~min}$ and then placed on a foam pad as opposed to being stereotactically fixed to allow for rapid acceleration and deceleration of the head upon impact of the weight. The analgesic buprenorphine was administered subcutaneously at $0.05-0.1 \mathrm{mg} / \mathrm{kg}$. The mouse's head was wetted with a cotton swab soaked in $70 \%$ ethanol. A flat, stainless steel disc $1.3 \mathrm{~cm}$ in diameter, $1 \mathrm{~mm}$ thick, and weighing 880 mg was then placed on the wetted head. The disc prevented skull breaks and diffused the impact across the entire cranium. The mouse on the foam block was then lined up under a vertical Plexiglas tube containing a $100 \mathrm{~g}$ weight suspended via a pin at 50,60 , or $70 \mathrm{~cm}$ above the metal disc. To initiate the impact, the pin was pulled, allowing the weight to free fall and injure the mouse. The mouse was then placed on a sterile convertertopped heating pad on its back until consciousness resumed and the mouse righted itself. This time period is considered the righting reflex recovery time and was measured as a surrogate for loss of consciousness (LOC). All results except for Figure 1 used three impacts from $50 \mathrm{~cm}$ height with $45 \mathrm{~min}$ intervals causing repetitive diffuse TBI (rdTBI) because this paradigm minimized differences between mouse strains and 
Table 1. Manufacturer information, working dilutions and resource identification numbers for primary and secondary antibodies used for immunohistochemistry

\begin{tabular}{|c|c|c|c|c|c|c|}
\hline Name & Manufacturer & Catalog \# & RRID & Species raised in & Monoclonal/polyclonal & Concentration \\
\hline \multicolumn{7}{|l|}{ Primary antibodies } \\
\hline GFP & Aves Lab & GFP-1020 & AB_300798 & Chicken & Polyclonal & $1: 1000$ \\
\hline GFAP & Millipore & MAB360 & AB_2109815 & Mouse & Monoclonal & $1: 1000$ \\
\hline GFAP & Dako & Z0334 & AB_10013382 & Rabbit & Polyclonal & $1: 1000$ \\
\hline GFAP & Abcam & AB4674 & AB_304558 & Chicken & Polyclonal & $1: 1000$ \\
\hline Vimentin & Millipore & AB5733 & AB_1121377 & Chicken & Polyclonal & $1: 1000-1: 5000$ \\
\hline Nestin & Millipore & MAB353 & AB_94911 & Mouse & Monoclonal & $1: 200$ \\
\hline Glt1 & Millipore & AB1783 & AB_90949 & Guinea Pig & Polyclonal & $1: 1000$ \\
\hline Kir4.1 & Alomone & APC035 & AB_2040120 & Rabbit & Polyclonal & $1: 400$ \\
\hline Cleaved Caspase 3 & Cell Signaling Technology & 9661 & $A B \_2341188$ & Rabbit & Polyclonal & $1: 100$ \\
\hline $\mathrm{S} 100 \beta$ & Sigma-Aldrich & $\mathrm{S} 2532$ & AB_477499 & Mouse & Monoclonal & $1: 1000$ \\
\hline$S 100$ & Dako & 0803 & Not assigned & Rabbit & Polyclonal & $1: 1000$ \\
\hline lba1 & Wako & $019-9741$ & $A B \_839504$ & Rabbit & Polyclonal & $1: 1000$ \\
\hline NeuN & Millipore & MAB377 & AB_2298772 & Mouse & Monoclonal & $1: 200$ \\
\hline NeuN & Millipore & ABN78 & AB_10807945 & Rabbit & Polyclonal & $1: 500$ \\
\hline CD11b & Bio-Rad & MCA74G & AB_321293 & Rat & Monoclonal & $1: 500$ \\
\hline Ki-67 & Thermo Scientific & RM-9106-S1 & AB_149792 & Rabbit & Monoclonal & $1: 100$ \\
\hline BrdU & Abcam & AB6326 & $A B \_305426$ & Rat & Monoclonal & $1: 500$ \\
\hline Anti-human tau & Thermo Scientific & MN1000B & $A B \_223453$ & Mouse & Monoclonal & $1: 100$ \\
\hline \multicolumn{7}{|l|}{ Secondary antibodies } \\
\hline Chicken Alexa Fluor-488 & Jackson Immuno Research & $703-546-155$ & AB_2340376 & Donkey & Polyclonal & $1: 1000$ \\
\hline Chicken Cy3 & Jackson Immuno Research & $703-166-155$ & $A B \_2340364$ & Donkey & Polyclonal & $1: 1000$ \\
\hline Chicken Alexa Fluor-647 & Jackson Immuno Research & $703-606-155$ & AB_2340380 & Donkey & Polyclonal & $1: 1000$ \\
\hline Rabbit Alexa Fluor-488 & Jackson Immuno Research & $111-546-144$ & $A B \_2338057$ & Goat & Polyclonal & $1: 1000$ \\
\hline Rabbit Cy3 & Jackson Immuno Research & $111-166-144$ & AB_2338011 & Goat & Polyclonal & $1: 1000$ \\
\hline Rabbit Alexa Fluor-647 & Jackson Immuno Research & $111-606-144$ & $A B \_2338083$ & Goat & Polyclonal & $1: 1000$ \\
\hline Mouse Alexa Fluor-488 & Jackson Immuno Research & $115-546-003$ & $A B \_2338859$ & Goat & Polyclonal & $1: 1000$ \\
\hline Mouse $\mathrm{Cy} 3$ & Jackson Immuno Research & $115-166-003$ & $A B \_2338699$ & Goat & Polyclonal & $1: 1000$ \\
\hline Mouse Alexa Fluor-647 & Jackson Immuno Research & $115-606-003$ & AB_2338921 & Goat & Polyclonal & $1: 1000$ \\
\hline Rat Alexa Fluor-488 & Jackson Immuno Research & $112-546-003$ & $A B \_2338364$ & Goat & Polyclonal & $1: 1000$ \\
\hline Rat Cy3 & Jackson Immuno Research & $112-166-003$ & AB_2338253 & Goat & Polyclonal & $1: 1000$ \\
\hline Rat Alexa Fluor-647 & Jackson Immuno Research & $112-606-003$ & $A B \_2338406$ & Goat & Polyclonal & $1: 1000$ \\
\hline Guinea pig Alexa Fluor-488 & Jackson Immuno Research & $106-546-003$ & $A B \_2337441$ & Goat & Polyclonal & $1: 1000$ \\
\hline Guinea pig Cy3 & Jackson Immuno Research & $106-166-003$ & $A B \_2337426$ & Guinea pig Cy3 & Polyclonal & $1: 1000$ \\
\hline Guinea pig Alexa Fluor-647 & Jackson Immuno Research & $106-606-003$ & $A B \_2337449$ & Goat & Polyclonal & $1: 1000$ \\
\hline \multicolumn{7}{|l|}{ Dyes } \\
\hline DAPI & Thermo Fisher & D1306 & AB_2629482 & $\mathrm{N} / \mathrm{A}$ & $\mathrm{N} / \mathrm{A}$ & $1: 1000$ \\
\hline Alexa Fluor-555 cadaverine & Thermo Fisher & A30677 & $\mathrm{N} / \mathrm{A}$ & $\mathrm{N} / \mathrm{A}$ & $N / A$ & $0.33 \mathrm{mg} / \mathrm{mouse}$ \\
\hline NeuroTrace 530/615 Nissl & Thermo Fisher & N21482 & AB_2620170 & $\mathrm{N} / \mathrm{A}$ & $\mathrm{N} / \mathrm{A}$ & 1:100 \\
\hline
\end{tabular}

N/A, Not applicable.

sexes and was characterized by low mortality rates (see "Diffuse mild/ concussive TBI induced LOC but no structural lesions or tissue loss" section in Results).

\section{Histological procedures}

Mice were deeply anesthetized with ketamine and xylazine before transcardial perfusion with PBS followed by $4 \%$ paraformaldehyde (PFA). Brain tissue was collected and postfixed in 4\% PFA overnight. Coronal slices were cut at $40-50 \mu \mathrm{m}$ thickness using a vibratome (Campden $5100 \mathrm{mz}$ ). Immunohistochemistry was performed using primary antibodies as specified in Table 1 in PBS with $0.5 \%$ Triton X-100 and 10\% goat serum at $4^{\circ} \mathrm{C}$ overnight. Slices were washed in PBS and incubated in secondary antibodies (Table 1 ) in $1 \times$ PBS with $0.5 \%$ Triton X-100 and $10 \%$ goat serum for $1-2 \mathrm{~h}$ at room temperature. 4,6-diamidino-2phenylindole (DAPI) was included in the secondary antibody solution. Slices were washed in PBS and mounted on glass microscope slides using Aqua Poly/Mount (Polysciences, catalog \#18606).

Nissl staining followed immunohistochemistry before the slices were mounted on glass slides. After the secondary antibody incubation period, slices were washed in PBS for $10 \mathrm{~min}$ and placed in PBS with $0.5 \%$ Triton $\mathrm{X}-100$ to allow the tissue to permeabilize for $10 \mathrm{~min}$, followed by a $10 \mathrm{~min}$ wash in PBS. Slices were incubated in 1:100 NeuroTrace (Table 1) in PBS for $20 \mathrm{~min}$. After incubation, the slices were washed in PBS with $0.5 \%$
Triton X-100 for $10 \mathrm{~min}$, followed by two $10 \mathrm{~min}$ washes in PBS. Slices were mounted on glass slides and embedded using Aqua Poly/Mount.

Images of sham and rdTBI mice were acquired using a Nikon A1R confocal microscope with Nikon $4 \times, 10 \times$, or $20 \times$ air objectives or Nikon Apo $40 \times / 1.30$ and $60 \times / 1.40$ oil-immersion objectives.

\section{Western blotting}

Adult male and female mice that incurred rdTBI were killed by cervical dislocation at $4 \mathrm{~h}$ postinjury (hpi), $3 \mathrm{~d}$ postinjury (dpi), $7 \mathrm{dpi}$, or $14 \mathrm{dpi}$. Sham animals were killed at the $4 \mathrm{~h}$ ( 9 animals) or $7 \mathrm{~d}$ time point (3 animals). Time points reflect the time period after the initial injury; that is, the $4 \mathrm{hpi}$ time point was collected $4 \mathrm{~h}$ after the first impact and $2.5 \mathrm{~h}$ after the last impact. Cortical gray matter and hippocampus were rapidly dissected, snap frozen on dry ice, and stored at $-80^{\circ} \mathrm{C}$. Tissues were mechanically homogenized in radioimmunoprecipitation assay buffer with 1:100 protease and phosphatase inhibitors (Sigma-Aldrich, catalog \#P8340, P0044), followed by sonication ( $3 \times 10 \mathrm{~s}$ pulse/rest $5 \mathrm{~s}, 30 \%$ amplitude) on ice. Remaining tissue fragments were spun down and protein supernatants were stored at $-80^{\circ} \mathrm{C}$. Protein concentrations were determined for each sample using a bicinchoninic acid assay. $15 \mu \mathrm{g}$ of protein was mixed with sample buffer $(4 \times$ Laemmli sample buffer with $\beta$-mercaptoethanol) and 1:10 dithiothreitol, denatured at $95^{\circ} \mathrm{C}$ for 10 min, and loaded into Bio-Rad Criterion TGX precast 26-well gels. Gels 
Table 2. Manufacturer information, working dilutions, and resource identification numbers for primary and secondary antibodies used for Western blot

\begin{tabular}{|c|c|c|c|c|c|c|}
\hline Name & Manufacturer & Catalog\# & RRID & Species raised in & Monoclonal/polyclonal & Concentration \\
\hline \multicolumn{7}{|l|}{ Primary antibodies } \\
\hline GFAP & Dako & 20334 & AB_10013382 & Rabbit & Polyclonal & 1:5000 \\
\hline Vimentin & Millipore & AB5733 & $A B \_11212377$ & Chicken & Polyclonal & $1: 5000$ \\
\hline Vimentin & Thermo Fisher & MA3-745 & $A B \_326285$ & Mouse & Monoclonal & $1: 5000$ \\
\hline Nestin & Abcam & AB6142 & $A B \_305426$ & Mouse & Monoclonal & $1: 2500$ \\
\hline Cleaved Caspase 3 & Cell Signaling Technology & 96115 & AB_2341188 & Rabbit & Polyclonal & $1: 1500$ \\
\hline GAPDH & Abcam & AB8245 & $A B \_2107448$ & Mouse & Monoclonal & $1: 5000$ \\
\hline \multicolumn{7}{|l|}{ Secondary antibodies } \\
\hline AzureSpectra goat anti-rabbit IgG IR800 antibody & Azure Biosystems & AC2134 & Not assigned & Goat & Polyclonal & $1: 1500$ \\
\hline AzureSpectra goat anti-mouse lgG IR700 antibody & Azure Biosystems & AC2129 & Not assigned & Goat & Polyclonal & $1: 1500$ \\
\hline AzureSpectra goat anti-mouse lgG IR800 antibody & Azure Biosystems & AC2135 & Not assigned & Goat & Polyclonal & 1:1500 \\
\hline AzureSpectra goat anti-rabbit IgG IR700 antibody & Azure Biosystems & AC2128 & Not assigned & Goat & Polyclonal & $1: 1500$ \\
\hline AzureSpectra goat anti-chicken IgG IR700 antibody & Azure Biosystems & $A C 2131$ & Not assigned & Goat & Polyclonal & $1: 1500$ \\
\hline Goat anti-rabbit lgG HRP & Santa Cruz Biotechnology & SC2004 & $A B \_631746$ & Goat & Polyclonal & $1: 1500$ \\
\hline Goat anti-mouse lgG HRP & Santa Cruz Biotechnology & SC2055 & $A B \_631738$ & Goat & Polyclonal & $1: 1500$ \\
\hline Goat anti-chicken IgG HRP & Santa Cruz Biotechnology & SC2901 & AB_650515 & Goat & Polyclonal & $1: 1500$ \\
\hline \multicolumn{7}{|l|}{ Chemiluminescent substrates } \\
\hline Name & Manufacturer & Catalog\# & & \multicolumn{2}{|c|}{ Development conditions } & Concentration \\
\hline Clarity Western ECL substrate-peroxide solution & Bio-Rad & 102030831 & & \multicolumn{2}{|c|}{1 min incubation, room temperature } & $1: 2$ \\
\hline Clarity Western ECL substrate-luminol/enhancer solution & Bio-Rad & 102030829 & & \multicolumn{2}{|c|}{1 min incubation, room temperature } & $1: 2$ \\
\hline
\end{tabular}

N/A, Not applicable.

were run at $80 \mathrm{~V}$ until dye line narrowed and then at $200 \mathrm{~V}$ for $\sim 30-45$ min. The Trans-blot Turbo Transfer system was used for semidry transfer at $2.5 \mathrm{~A}$ and $25 \mathrm{~V}$ for $10 \mathrm{~min}$. Membranes were blocked in 5\% nonfat milk (NFM) in Tris-buffered saline-Tween 20 (TBST) for 30 min. Membranes were incubated in primary antibody solution (Table 2) in 5\% NFM/TBST overnight at $4^{\circ} \mathrm{C}$. Membranes were washed and incubated in secondary antibody solution in 5\% NFM/TBST for $1 \mathrm{~h}$ at room temperature. Membranes were developed either with luminol/peroxide developer solution (Bio-Rad) or imaged directly after incubation with near infrared secondary antibodies. For the latter, following transfer membranes were instead blocked for 10 min in Azure fluorescent blocking buffer (Azure Biosystems). Membranes were incubated in primary antibody solution in Azure fluorescent blocking buffer overnight at $4^{\circ} \mathrm{C}$. Membranes were washed in Azure IR-Fluorescent washing solution (Azure Biosystems) and incubated in secondary antibody solution in Azure fluorescent blocking buffer for $1 \mathrm{~h}$ at room temperature. Membranes were washed in PBS and immediately imaged with the Azure c500 imaging system.

\section{Two-photon imaging through a thinned-skull window}

Twelve- to 16-week-old Aldh1l1-eGFP//FVB/N mice of both sexes were used to image astrocytes over time and, importantly, before and after TBI. In this mouse line, astrocyte cell bodies can be identified easily, whereas fine processes are harder to detect when in vivo two-photon imaging was performed through a thinned-skull window. Before surgery, mice were anesthetized with $3 \%$ isoflurane gas in an induction chamber and administered analgesics $(0.1 \mathrm{mg} / \mathrm{kg}$ buprenorphine, Par Pharmaceuticals) and the nonsteroidal anti-inflammatory rimadyl/carprofen subcutaneously ( $5 \mathrm{mg} / \mathrm{kg}$, Zoetis Services). During the surgery, the animal's head was fixed in a stereotactic apparatus and the body temperature was maintained at $37.0^{\circ} \mathrm{C}$. The level of anesthesia was maintained at $1.5-2 \%$ isoflurane gas during the surgery. An eye lubricant was applied to the eyes of the animal to prevent corneal desiccation. A circular area of the skull (typically $\sim 2 \mathrm{~mm}$ in diameter) over the region of interest (ROI) was shaved using a hand drill. The ROI was thinned to $\sim 50 \mu \mathrm{m}$ thickness (allowing for imaging $\sim 100 \mu \mathrm{m}$ deep into the cortex). Dental cement was used to cover the area around the ROI and to create a well. Mice were placed into a warm clean cage with hydrogel and moistened food to recover. After $1 \mathrm{~h}$, baseline images were acquired using a four-channel multiphoton laser scanning fluorescence microscope (Olympus FV1000MPE) equipped with an XLPLN25X/1.05 numerical aperture (NA) water-immersion objective (Olympus). During the imaging session, mice were lightly anesthetized using isoflurane to alleviate stress from restraining their head in a stereotactic apparatus (two-photon imaging is not painful after the cranial window has been prepared and mice recovered from this surgery). Mouse body temperature was maintained at $37.0^{\circ} \mathrm{C}$. After the baseline imaging session, mice were allowed to recover for $1 \mathrm{~h}$. After the recovery period, mice were anesthetized with 3\% isoflurane gas in an induction chamber for $5 \mathrm{~min}$ and underwent removal of the dental cement cap. A metal disc was then placed over the exposed skull and rdTBI was induced 3 times with 45 min intervals between impacts. After the final impact, mice were placed in the stereotactic apparatus to apply cyanoacrylate glue (Henkel) and cover the ROI with a cover glass ( $\# 0$, thickness: $0.10 \pm 0.02 \mathrm{~mm}$, diameter $3 \mathrm{~mm}$, Fisher Scientific, 22-031145), thereby installing a permanent window for longterm reimaging. During the imaging window installation, mice were kept at $1.5-2 \%$ isoflurane gas level. Dental cement was applied to create a well and cover the area around the thinned ROI. The control mouse underwent only thinned skull preparation surgery, cover glass implantation, and imaging.

\section{Video-EEG data acquisition}

Epidural recordings were obtained from 12- to 16-week-old male C57BL/6 mice. Mice were anesthetized and positioned in the weightdrop device (as described in the "Impact acceleration (weight drop) injury" section) and received either three TBIs or three sham (3xSham) injuries with 45 min intervals between impacts/sham. Mice were allowed to recover for at least $1 \mathrm{~h}$ after the last TBI before surgery. Then, mice were anesthetized with $3 \%$ isoflurane gas and positioned in the stereotactic apparatus (Kopf Instruments). Surgery was performed using surgical tools sterilized by autoclaving before surgery and resterilizing in a dry sterilizer (Germinator 500, Cellpoint Scientific) for $3 \mathrm{~min}$ in between animals. For EEG acquisition, we either implanted bipolar intracranial electrodes connected to a plastic pedestal (Plastics One) positioned in the parietal area over each hemisphere with a ground electrode placed over the cerebellum or a circuit board (Pinnacle Technology) configured to obtain both EEG and EMG activity. The latter was affixed to the skull using skin glue and 4 stainless steel intracranial screws, covered with silver epoxy. Electromyography (EMG) was recorded using two stainless steel subdermal electrodes overlying the nuchal muscles. Dental acrylic cement was used to cover the electrodes and skull. The scalp incision was then closed with skin glue. When intracranial electrodes (Plastics One) were used, two additional stainless steel screws were anchored into the skull to provide stability for the dental acrylic, applied to cover the electrodes and the screws. After surgery, mice were placed in individ- 
ual cages and warmed on the heating pad $\left(31-33^{\circ} \mathrm{C}\right)$ for at least $1 \mathrm{~h}$ with clean bedding, recovery gel, and moistened food. Next, mice were placed into the individual Plexiglas video-EEG monitoring cylinders with fresh bedding, dry food, recovery gel, water bottles, and nesting material for acclimatization before long-term video-EEG recording. Mice were allowed to recover $4-7 \mathrm{~d}$ after the TBI and surgery. Continuous 24/7 video-EEG was then obtained for up to $110 \mathrm{dpi}$ using either Pinnacle Technology or Biopac EEG acquisition hardware and software. In two sham mice and three mice with convulsive seizures, video recording was acquired starting on day 39 and 35 postsurgery, respectively; in all other mice, video and EEG recordings started on the same day. Animal behavior was recorded with individual box or dome cameras fully synchronized with the EEG recording. EEG data were acquired at $500 \mathrm{~Hz}$ (Biopac Systems) or 400-1000 Hz sampling rates (Pinnacle Technology) and filtered (high-pass filter cutoff: $0.05 \mathrm{~Hz}$; Biopac Systems) or $1 \mathrm{~Hz}$ (Pinnacle Technology) (low-pass filter cutoff: $100 \mathrm{~Hz}$ in both systems). Cameras had either integrated or separate infrared sources for dark hours video acquisition and were affixed over (dome cameras) or in front (box cameras) of the Plexiglas cylinders. All animals were kept in a standard $12 \mathrm{~h}$ light/12 h dark cycle.

\section{Quantification of astrocyte coupling using biocytin filling}

Aldh1l1-tdTomato animals between 8 and 12 weeks of age were anesthetized with $3 \%$ isofluorane and subjected to either rdTBI or $3 x$ Sham as described above. Animals were killed by cervical dislocation at 7-15 dpi and $300 \mu \mathrm{m}$ acute coronal brain slices were cut using a compresstome (Precisionary Instruments) in ice-cold cutting solution containing the following (in mM): $135 \mathrm{~N}$-methyl-D-gluconate, $1.5 \mathrm{KCl}, 1.5 \mathrm{KH}_{2} \mathrm{PO}_{4}, 23$ choline bicarbonate, $25 \mathrm{D}$-glucose, $0.5 \mathrm{CaCl}_{2}$, and $3.5 \mathrm{MgSO}_{4}$. After recovery in artificial CSF (containing the following in mM: $125 \mathrm{NaCl}, 3 \mathrm{KCl}$, $1.25 \mathrm{NaH}_{2} \mathrm{PO}_{4}, 25 \mathrm{NaHCO}_{3}, 2 \mathrm{CaCl}_{2}, 1.3$ or $2 \mathrm{MgSO}_{4}$, and $25 \mathrm{D}$-glucose) at $37^{\circ} \mathrm{C}$ for $30 \mathrm{~min}$, slices were maintained at room temperature until the start of the experiment. Aldh1l1-tdTomato ${ }^{+}$cells (astrocytes) were identified in cortical layer 1 or 2 , patched, and filled with a $5 \mathrm{mg} / \mathrm{ml}$ biocytin solution for $20 \mathrm{~min}$ at $37^{\circ} \mathrm{C}$ and then the slice was fixed in $4 \%$ PFA for at least $2 \mathrm{~h}$. After fixation, slices were rinsed in PBS and stained with Glt-1 antibody overnight. After three washes in PBS, the slices were incubated with a secondary antibody conjugated to Alexa Fluor 647, DAPI, and streptavidin conjugated to Alexa Fluor 488 for $1 \mathrm{~h}$ at room temperature, washed in PBS, and mounted with Aqua Poly/Mount (Polysciences, catalog \#18606). Slices were imaged using a Nikon A1R confocal microscope using $20 \times$ and $40 \times$ objectives.

\section{Data analysis}

Unbiased stereology. Quantification of the area covered by glial fibrillary acidic protein-positive $\left(\mathrm{GFAP}^{+}\right)$or Aldh1l1-eGFP ${ }^{+}$astrocytes as well as Glt1-negative area was performed using unbiased stereology (MBF Bioscience Stereo Investigator) on an Axiovert $200 \mathrm{M}$ inverted microscope (Carl Zeiss) using the $20 \times / 0.75 \mathrm{NA}$ objective on five $40 \mu \mathrm{m}$ coronal slices of Aldh1l1-eGFP mice that were labeled with antibodies against GFP, GFAP, and Glt1. The cortical gray matter (with exception of the piriform cortex) was outlined using the $10 \times$ objective. We chose extensive sampling since areas that contained $\mathrm{GFAP}^{+}$astrocytes or lacked Aldh1l1$\mathrm{eGFP}^{+}$and Glt1 signal, were small. The counting frame was $300 \mu \mathrm{m} \times$ $300 \mu \mathrm{m}$, grid size was set to $600 \mu \mathrm{m} \times 600 \mu \mathrm{m}$. Guard zones were set to $5 \mu \mathrm{m}$ at both edges of the slice $z$-stack. The combination of counting frame size and grid frame size was chosen after a pilot study was performed analyzing the same sample with three different grid sizes 300 $\mu \mathrm{m} \times 300 \mu \mathrm{m}$ (sampling 100\% of the area), $600 \mu \mathrm{m} \times 600 \mu \mathrm{m}$ (sampling $25 \%$ of the area), and $750 \mu \mathrm{m} \times 750 \mu \mathrm{m}$ (sampling $16 \%$ of the area). There was no significant difference between the $600 \mu \mathrm{m} \times 600$ and $300 \mu \mathrm{m} \times 300 \mu \mathrm{m}$ measurements, but $750 \mu \mathrm{m} \times 750 \mu \mathrm{m}$ resulted in a discrepancy larger than the approximated effect size.

Quantification of Glt1 loss area in EEG-recorded animals. We used three to five slices per animal to assess the cortical area covered with $\mathrm{GFAP}^{+}$or S100 $\beta^{+}$astrocytes and Glt1. Images were acquired using a Nikon A1R microscope with a $10 \times$ air objective from $40 \mu \mathrm{m}$ coronal slices from C57BL6 mice labeled with antibodies against GFAP or S100 $\beta$, and Glt1. The cortical gray matter (with exception of the piriform cortex) was outlined using a freehand selection tool in ImageJ. This area was measured for each slice. Similarly, areas with downregulation of Glt1 were manually outlined, measured, and expressed as fractions of the total area for each slice. The values of Glt1 area fraction loss were then averaged between slices for each animal.

Quantification of astrocyte proliferation. Astrocyte proliferation was assessed using either immunohistochemistry against the cell cycle protein Ki67 at 3 dpi or bromodeoxyuridine (BrdU) labeling at 7 dpi. For the latter, rdTBI or $3 \mathrm{xSham}$ mice received intraperitoneal injections of $\mathrm{BrdU} /$ saline ( $50 \mu \mathrm{g} / \mathrm{g}$ body weight) twice daily for $7 \mathrm{~d}$ starting on the day of rdTBI/Sham. To quantify the number of $\mathrm{Ki} 67^{+}$astrocytes, three confocal images were taken in four different slices per animal, three animals per experimental group, in ROIs with loss of Aldh1l$\mathrm{eGFP}^{+}$and ROIs with $\mathrm{GFAP}^{+}$astrocytes. To quantify the number of $\mathrm{BrdU}^{+}$astrocytes, one or two confocal images were taken in three different slices per animal, three animals per experimental group, in ROIs with loss of Aldh1l-eGFP ${ }^{+}$and ROIs with GFAP ${ }^{+}$astrocytes. Confocal images had a $z$-step size of $1 \mu \mathrm{m}$ and stacks spanned the entire slice and were quantified step by step using the cell counter tool in ImageJ. Cells that colabeled for GFP/DAPI and Ki67 or BrdU were considered proliferating astrocytes.

Quantification of astrocyte coupling using biocytin filling. From a total of 43 slices, the ones with the biocytin injected adjacent to an area with dysfunctional astrocytes were selected $(n=18)$. Size of areas with atypical astrocytes was detected using the binarization and image calculator tool in ImageJ based on the presence of tdTomato $\left(\operatorname{tdTom}^{+}\right)$and the absence of Glt1 $\left(\mathrm{Glt1}^{-}\right)$. For neighboring non-atypical astrocytes, area size was determined based on the presence of both labels (consistent settings between slices). Next, using the image calculator, the percentage of biocytin-positive pixels in tdTomato ${ }^{+}$and $\mathrm{Glt}^{+}{ }^{+}$and atypical areas $\left(\mathrm{TdTom}^{+} / \mathrm{Glt}^{-}{ }^{-}\right)$were calculated and corrected by area size. If biocytin were evenly distributed between atypical areas and TdTom ${ }^{+} / \mathrm{Glt}^{+}{ }^{+}$area, then each area would have an expected value of $50 \%$ biocytin pixels and both areas would add up to a total value of $100 \%$.

Quantification of neuronal densities. Nissl and NeuN was stained for in $3 \mathrm{xSham}$ tissue ( $n=4$ for $1,3,7$, and $14 \mathrm{~d}$ each) and rdTBI tissue $(n=5$ for $1 \mathrm{dpi}, n=3$ for $3 \mathrm{dpi}, n=7$ for $7 \mathrm{dpi}, n=4$ for $14 \mathrm{dpi}$, and $n=3$ for $28 \mathrm{pdi}$ ) and then imaged using confocal microscopy. The area of regions with astrocyte reporter loss, as well as the surrounding region (periphery), was quantified using ImageJ. From this area, the density of the $\mathrm{Nissl}^{+}$neurons in cortical layers II/III was determined using the cell counter tool in ImageJ. Neuronal densities in peripheral areas were set to 1 and the fold change of densities within areas of astrocytic downregulation was normalized accordingly for each ROI.

Quantification of Western blot data. Blots were quantified with Azurespot analysis software. Images were converted to grayscale for band quantification. Lane detection software prevented lane overlap and accounted for the curvature of some lanes. Rolling ball background subtraction was used for each blot image. Automatic band detection was first used to detect band peak gray values, followed by manual adjustment to restrict the area of band measurement to the band edges. Gray values of each band were measured in the above-defined frames. For GFAP, the 55 $\mathrm{kDa}$ and $48 \mathrm{kDa}$ bands were measured in one frame because they ran close together. For Ibal, the reported band of $17 \mathrm{kDa}$ was measured. Band gray values were divided by band gray values of a GAPDH loading control to determine relative protein levels for each sample. One sham value closest to the average of all sham values was used to normalize the gray values on each blot.

Video-EEG data analysis. Digital EEG files were analyzed offline manually by browsing the entire file on the computer screen and then confirmed using semiautomatic analysis software (DClamp, Pediatric Epilepsy Research, Massachusetts General Hospital). A synchronized video recording was then used to associate a behavioral component with convulsive or nonconvulsive seizures and to differentiate from movement artifacts and signal noise. An electrographic seizure was defined as high-amplitude rhythmic discharges with the following epileptiform characteristics: (1) an evolution of the ictal event in amplitude and frequency; (2) a defined EEG pattern of oscillations within an ictal event: spikes, polyspikes, spike-and-wave discharges, 
or slow waves; (3) the seizure duration minimum $10 \mathrm{~s}$; (4) epileptiform (interictal) spiking typically emerging before the onset of the seizure; and (5) the ictal event followed by postictal depression (sudden EEG background suppression). Nonconvulsive seizure was defined as sustained rhythmic epileptiform electrographic activity accompanied by alterations in behavior in the absence of convulsive movements (Mader et al., 2012). Power spectrum density was analyzed in MATLAB (The MathWorks) for both convulsive and nonconvulsive seizures in rdTBI animals.

\section{Experimental design and statistics}

Animals were distributed randomly into experimental groups. For each experiment, groups included "TBI" and "Sham." Figures 2, 3, 4, 5, 6, 7 , and 8 assessed outcome measures along a timeline after TBI/Sham. Figure 1 assessed the variables mouse strain and TBI/Sham for outcome measures mortality and LOC. Figures 1, 2, and 3 assessed the variables sex and TBI/Sham for outcome measures mortality, LOC, GFAP upregulation, and Glt1 loss. Data were tested for normal distribution using the Kolmogorov-Smirnov normality test. The precise statistical tests used for each analysis were chosen as appropriate and specified in the Results section and in Extended Figures 1-1 (available as Extended Data), 2-1 (available as Extended Data), 3-1 (available as Extended Data), 4-1 (available as Extended Data), 5-1 (available as Extended Data), 6-1 (available as Extended Data), 7-1 (available as Extended Data), and 8-1 (available as Extended Data) (typically two-way ANOVA with post hoc analysis for differences between multiple groups). Unless stated otherwise, all values are reported as mean with SEM.

Statistics were computed and graphed with GraphPad Prism 7 software. The significance level was set to $p \leq 0.05$. Scatter plots display individual values and bar graphs display the mean with SEM. Statistical significance is indicated with ${ }^{*} p \leq 0.05,{ }^{* *} p \leq 0.01,{ }^{* *} p \leq 0.001$, ${ }_{* * * *} p \leq 0.0001$.

\section{Biological variables}

Parts of our histological analysis relied on the use of transgenic astrocyte reporter mice (Aldh1l1-eGFP) on FVB/N background, but C57BL/6 mice are more widely used in TBI and epilepsy research. Therefore, we assessed mortality rates, LOC, and histology in both strains. Females are traditionally excluded from TBI research based on studies showing a neuroprotective effect of endogenous estrogen levels potentially increasing biological variability and complicating data interpretation. Rather than excluding one sex, we assessed whether male and female mice responded differently to our injury paradigm (see Figs. 1, 2, 3).

Single and repetitive TBI caused similar astrocyte abnormalities in both sexes (further described below), albeit at a lower frequency after single injury. Given that these effects were mild even after repetitive injury, we only assessed rdTBI animals in further detail. Both sexes were included in subsequent experiments and analyzed separately unless otherwise specified. Samples from male and female mice were pooled for histological analysis. Animal sex for these samples is specified in Figures 1-1 (available as Extended Data), 2-1 (available as Extended Data), 3-1 (available as Extended Data), 4-1 (available as Extended Data), 5-1 (available as Extended Data), 6-1 (available as Extended Data), 7-1 (available as Extended Data), and 8-1 (available as Extended Data).

\section{Results}

\section{Diffuse mild/concussive TBI induced LOC but no structural lesions or tissue loss}

To induce diffuse TBI in the absence of focal injury, we chose an impact acceleration model calibrated to mild/concussive TBI (Foda and Marmarou, 1994; Marmarou et al., 1994, 2009; Nichols et al., 2016) in adult mice of both sexes at 12-16 weeks of age. Our criteria for mild/concussive TBI included the following: (1) a significantly prolonged period of unconsciousness of injured mice compared with Sham mice, (2) a cumulative effect when injuries were repeated, (3) a lack of obvious tissue lesions and lack of tissue necrosis or loss, and (4) low mortality rates in accor- dance with the definition of mild TBI in humans (Management of Concussion/mTBI Working Group, 2009). In short, a $100 \mathrm{~g}$ weight guided by a Plexiglas tube was allowed to impact a metal disc placed on the mouse head while the mouse rested on a foam pad rather than being fixed in a stereotactic apparatus. This allowed for rapid acceleration and deceleration of the head upon impact inducing diffuse injury (Foda and Marmarou, 1994; Marmarou et al., 1994, 2009). Sham animals underwent the same procedure as the TBI mice (anesthesia, placement under the Plexiglas tube, etc.) with exclusion of an impact.

We first assessed different drop heights and interinjury intervals suitable to induce mild diffuse TBI in C57BL/6 and FVB/N mouse strains. Behavioral abnormalities consistent with those observed in patients with mild TBI were reported previously using a $100 \mathrm{~g}$ weight at $70 \mathrm{~cm}$ height (Nichols et al., 2016), which we used as a starting point.

\section{Mortality rates}

Single TBIs induced by weight drop from $50-70 \mathrm{~cm}$ height did not cause mortality in FVB/N or C57BL/6 mice (Fig. $1 a$ and below for strain and sex differences): 0/7 mice died after a single $70 \mathrm{~cm}$ impact. However, a $100 \mathrm{~g}$ weight dropped three times from $70 \mathrm{~cm}$ height ( $n=7 ; 24 \mathrm{~h}$ interinjury interval) or $60 \mathrm{~cm}$ height ( $n=38,24 \mathrm{~h}$ interinjury interval) resulted in mortality rates as high as $28.6 \%$ in $\mathrm{FVB} / \mathrm{N}$ mice $(70 \mathrm{~cm})$ and $29 \%$ in C57BL/6 mice $(60 \mathrm{~cm})$. This did not meet the criterion of a low mortality rate after repetitive TBI and we thus reduced the weight drop height to $50 \mathrm{~cm}$, which resulted in a mortality rates of 7\% in C57BL/6 mice ( $n=59$, males/females pooled, see Fig. $1 a$ for separate analysis) and $2 \%$ in FVB/N mice ( $n=64$, males/females pooled, see Fig. $1 a$ for separate analysis) even when injuries were spaced apart by only $45 \mathrm{~min}$.

A stepwise Bayesian information criterion was used to select a model that predicted mortality based on sex, weight, height of weight drop, interval between injuries, strain, or interactions between all predictors (Zhang, 2016). The presence of the injury (as opposed to no injury in Shams) was the best predictor when Sham animals were included in the analysis (confidence: $99.99 \%$ ). Height was the best predictor for mortality in animals with single and repetitive injuries when Sham animals were excluded from the analysis (confidence: $99.96 \%)$. Sex, weight, and strain were not significant predictors of mortality (for sample sizes of all groups, see Fig. 1-1, available as Extended Data).

\section{LOC and cumulative effect of repetitive TBIs}

In rodents, suppression of the righting reflex is a simple measure for unconsciousness (Morehead et al., 1994). Isoflurane anesthesia induced a short period of unconsciousness even in uninjured Sham controls (Fig. $1 b, p<0.0001$ for variable injury severity, Kruskal-Wallis ANOVA with Dunn's multiple-comparisons test; for detailed statistical comparison between groups, see Fig. 1-1, available as Extended Data). All injury groups presented with significant longer times spent unconscious compared with Sham controls. Single TBIs from $60 \mathrm{~cm}$ height caused significantly prolonged LOC compared with single TBI from $50 \mathrm{~cm}$. Importantly, repeating $50 \mathrm{~cm}$ TBIs three times with a 45 min interinjury interval doubled the time mice spent unconscious. Such a cumulative effect was not observed when TBIs were spaced $24 \mathrm{~h}$ apart even when the height was $60 \mathrm{~cm}$ (Fig. $1 b, p<0.0001$ for variable injury severity, Kruskal-Wallis ANOVA with Dunn's multiple-comparisons test, for statistical comparison between groups, see Fig. 1-1, available as Extended Data). A 
a Mortality rates (\%)

\begin{tabular}{|c|c|c|c|c|c|}
\hline \multirow{2}{*}{$\begin{array}{c}\text { Injury } \\
\text { Interval }\end{array}$} & \multirow[b]{2}{*}{ Injury } & \multicolumn{2}{|c|}{ Aldh1|1-eGFP } & \multicolumn{2}{|c|}{$\mathrm{C} 57 \mathrm{Bl} / 6$} \\
\hline & & Male & Female & Male & Female \\
\hline & 1x Sham & 0 & 0 & 0 & 0 \\
\hline & $1 \times$ TBI $50 \mathrm{~cm}$ & 0 & 0 & 0 & 0 \\
\hline & $1 \times$ TBI $60 \mathrm{~cm}$ & 0 & 이 & 0 & 0 \\
\hline \multirow{3}{*}{ 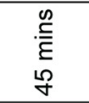 } & 3x Sham & 0 & 0 & 0 & 0 \\
\hline & $3 \times 50 \mathrm{~cm} \mathrm{TBI}$ & 3.1 & 0 & 8.3 & 5.7 \\
\hline & $3 \times 60 \mathrm{~cm} \mathrm{TBI}$ & 0 & 0 & n.a. & 66.7 \\
\hline \multirow{2}{*}{$\begin{array}{l}\text { 气 } \\
\text { ㅇ } \\
\text { ป }\end{array}$} & $3 \times$ Sham & 0 & 0 & 0 & 0 \\
\hline & $3 \times 60 \mathrm{~cm} \mathrm{TBI}$ & n.a. & n.a. & 12.5 & 33.3 \\
\hline
\end{tabular}

C

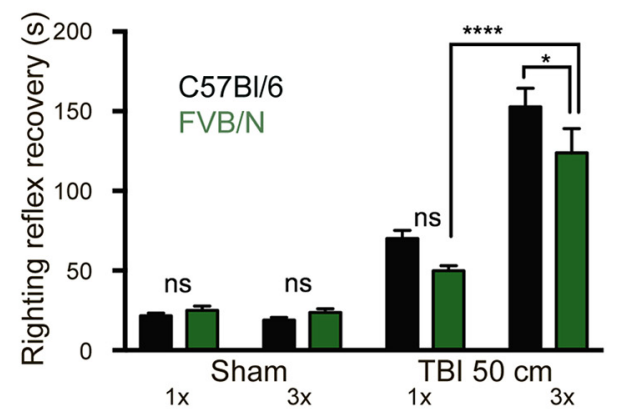

e

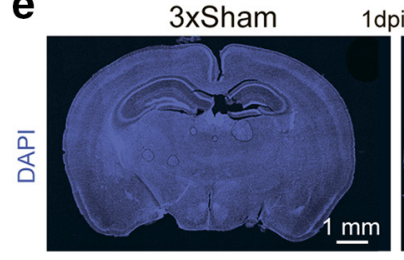

b

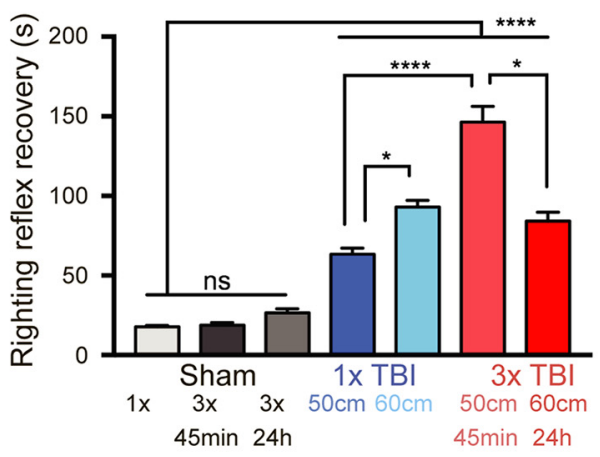

d
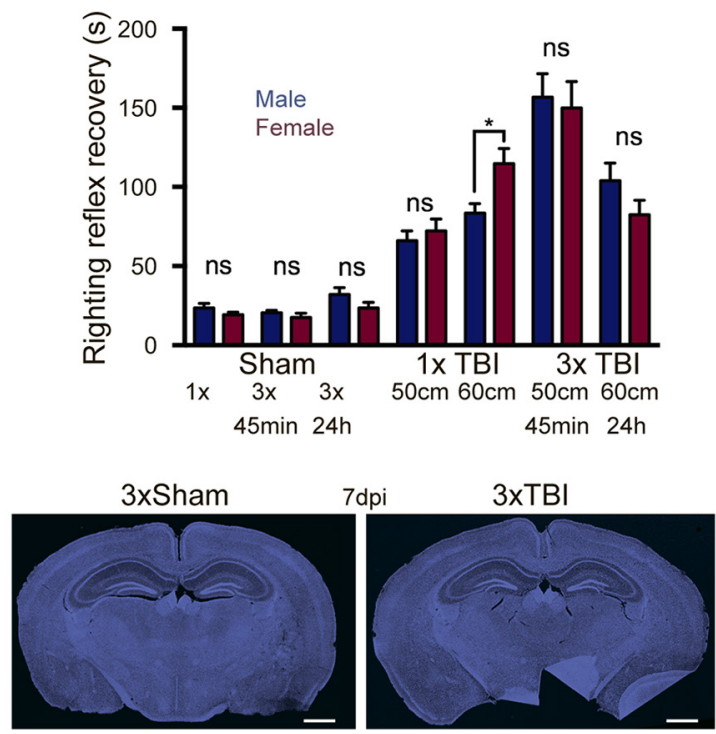

Figure 1. Mild/concussive TBI induced LOC but presented without obvious tissue damage in C $57 B L / 6$ and FVB/N mice. $a$, Males and females displayed similar mortality rates when the $3 \times 50 \mathrm{~cm}$ $45 \mathrm{~min}$ TBI paradigm was used. Mortality rates for $3 \times 60 \mathrm{~cm}$ injuries were high. $\boldsymbol{b}$, Increased injury severity and frequency as well as decreased interinjury intervals increased the length of the righting reflex recovery time as an indicator of $\mathrm{LOC}$ in $557 \mathrm{BL} / 6$ mice. $c$, $57 \mathrm{BL} / 6$ and FVB/N mice displayed comparable righting reflex recovery times after single TBI, but LOC was shorter in $\mathrm{FVB} / \mathrm{N}$ mice after rdTBI was induced. $\boldsymbol{d}$, Sex did not affect the righting reflex recovery time after single or repetitive mild/concussive TBI $(50 \mathrm{~cm}, 45 \mathrm{~min}$ interinjury interval) (rdTBI) in (57BL/6 mice. e, Histology of the tissue using DAPI staining showed that rdTBI did not cause obvious tissue damage or loss. See Figure 1-1 (available as Extended Data) for breakdown of sample sizes dependent on sex for representative images and detailed numerical data for all statistical group comparisons. ${ }^{*} p \leq 0.05,{ }^{* * *} p \leq 0.0001$.

small but significant difference after $50 \mathrm{~cm}, 45 \mathrm{~min}$ interinjury interval TBI between $\mathrm{C} 57 \mathrm{BL} / 6$ and $\mathrm{FVB} / \mathrm{N}$ mice was noted only when injuries were repeated (Fig. $1 c$, Fig. 1-1, available as Extended Data; 3xTBI $50 \mathrm{~cm} 45 \mathrm{~min}, p=0.0342$, Sidak's multiple-comparisons test; $p=0.1102$, two-way ANOVA for variable "strain/genetic background"). Righting reflex recovery times did not differ between C57BL/6 males and females after single or repetitive $50 \mathrm{~cm}$ TBI, but a significantly increased period of unconsciousness in females followed $60 \mathrm{~cm}$ single TBI (Fig. 1d, 1xTBI $60 \mathrm{~cm}, p=0.0124$, Sidak's multiplecomparisons test; $p=0.8702$, two-way ANOVA for variable "sex"; for sample sizes and statistical comparisons between groups, see Fig. 1-1, available as Extended Data).

\section{Lack of tissue lesions}

Last, we examined the brain tissue of mice injured once or repetitively ( $50 \mathrm{~cm}$ height, $45 \mathrm{~min}$ ) for tissue loss and large focal injury sites. We did not observe any tissue loss or large structural lesions in C57BL/6 $(n=25 \mathrm{TBI}$ animals $)$ or FVB/ $\mathrm{N}(n=61 \mathrm{TBI}$ animals $)$ mice in either male $(n=50 \mathrm{TBI})$ or female $(n=36 \mathrm{TBI})$ animals (Fig. 1e; for breakdown of sample size and sex of different injury paradigms, see Fig. 1-1, available as Extended Data). Similarly, when imaging tissue stained with hematoxylin and eosin, we did not observe any tissue abnormalities, including structural lesions. However, we occasionally found microscopic contusions characterized by enhanced cellular density surrounded by classic glial scar formation when the weight drop height was 60 or $70 \mathrm{~cm}$, but not in the $50 \mathrm{~cm}$ injury paradigm. Based on these data, we settled on an injury paradigm in which repetitive diffuse TBI is induced by three weight impacts dropped from a $50 \mathrm{~cm}$ height with a 45 min interinjury interval.

\section{rdTBI causes diffuse mild nonproliferative astrogliosis}

Increased expression of intermediate filaments, particularly of GFAP, is a standard indicator of astrogliosis. Immunohistochemistry against GFAP revealed small groups of astrocytes that upregulated GFAP in rdTBI mice $7 \mathrm{dpi}$. These groups were scattered across the cortical gray matter, a brain region that contains only few GFAP $^{+}$astrocytes in uninjured Sham controls (Fig. 2a). The area covered by GFAP ${ }^{+}$astrocytes was quantified using unbiased stereology at $7 \mathrm{dpi}$, a time point by which GFAP levels are at the peak after focal injuries, such as experimental stab wounds (Robel et al., 2011b). In the cortical gray matter (with exception 
of the piriform cortex), an area fraction of $20 \%( \pm 5$ SEM, $n=5$ ) was covered by GFAP ${ }^{+}$astrocytes after rdTBI, a fraction not significantly higher than in Sham controls (16.07 $\pm 3.18 \%$ SEM, $n=5$ for each group; $p=0.5251$, two-tailed unpaired $t$ test). However, the GFAP ${ }^{+}$main processes of astrocytes in rdTBI animals ( $n=3$ males) appeared thickened compared with Sham controls ( $n=6,3$ males/3females) (Fig. $2 b$ ).

To determine whether there is an overall change in GFAP protein expression, we next performed Western blot analysis within the cortical gray matter (Fig. $2 c$ ) or hippocampus (Fig. $2 h$ ) at different time points after rdTBI comparing male (Fig. 2e,j,g,n) and female mice (Fig. 2d, $k$; Fig. 2-1, available as Extended Data). In the cortical gray matter of females, GFAP protein expression was significantly increased at $4 \mathrm{hpi}(n=7, p=0.0192)$ and $14 \mathrm{dpi}$ $(n=5, p=0.0006)$ compared with Sham controls $(n=5$, Kruskal-Wallis ANOVA with Dunn's multiple-comparisons test; Fig. 2-1, available as Extended Data) (Fig. $2 c, d$ ). In females, the mean increase of GFAP protein at $7 \mathrm{dpi}$ appeared comparable to the 4 hpi and 14 dpi time points, yet only reached significance in a blot that directly compared males and females (Fig. $2 f, p=$ 0.0057, Sham vs females at $7 \mathrm{dpi}$, Kruskal-Wallis ANOVA with Dunn's multiple-comparisons test; for all group comparisons, see Fig. 2-1, available as Extended Data).

In the cortical gray matter of males, GFAP protein levels were increased significantly at $14 \mathrm{dpi}(n=5, p=0.0179$, Sham vs males at 14 dpi, Kruskal-Wallis ANOVA with Dunn's multiplecomparisons test; Fig. 2e; Fig. 2-1, available as Extended Data). Because we observed variability in the changes in GFAP levels after TBI between animals, a larger number of samples from males was tested at $7 \mathrm{dpi}$ and mean GFAP protein levels were significantly increased (Fig. $2 g)(n=16, p=0.0345$, Sham vs males at $7 \mathrm{dpi}$, unpaired, two-tailed $t$ test; Fig. 2-1, available as Extended Data). In summary, GFAP levels doubled by 7 dpi and tripled by $14 \mathrm{dpi}$ in both females and males.

In the hippocampus, where astrocytes express GFAP at higher levels in the uninjured brain, we did not observe an increase in GFAP levels at different time points after rdTBI in males (Fig. $2 h, j, p=0.5993$, Kruskal-Wallis with Dunn's multiplecomparisons test; Fig. 2-1, available as Extended Data), females (Fig. $2 k, p=0.4620$, One-way ANOVA $p=0.5993$, KruskalWallis with Dunn's multiple-comparisons test; Fig. 2-1, available as Extended Data), or when comparing both sexes (Fig. $2 m, p=$ 0.0438, Kruskal-Wallis with Dunn's multiple-comparisons test; Fig. 2-1, available as Extended Data). However, when the sample size was increased, a significant increase of GFAP after rdTBI was observed in males at $7 \mathrm{dpi}(n=13, p=0.0014)$ compared with sham controls $(n=6)$ (Fig. $2 n$, Mann-Whitney test, two-tailed, unpaired; Fig. 2-1, available as Extended Data).

Overall, upregulation of GFAP merely doubled or tripled as a result of rdTBI, which is relatively mild when considering the fivefold increase of GFAP after focal stab wounds (Vijayan et al., 1990), the sixfold increase in mouse brain tissue surrounding a glioma (Campbell et al., unpublished data), and the 16to 32-fold increase in Alexander's disease mouse models (Jany et al., 2013). Throughout all assays, we noted considerably high variability of GFAP protein levels from animal to animal after rdTBI.

\section{Astrocytes do not proliferate after rdTBI}

Mature astrocytes in the healthy adult brain are postmitotic, except for the astrocyte-like stem cells within adult neurogenic zones. After focal TBI, a subset of scar-forming astrocytes reenter the cell cycle to increase astrocyte numbers at the site of injury
(Buffo et al., 2008; Robel et al., 2011b). We assessed whether $\mathrm{GFAP}^{+}$astrocytes proliferated after rdTBI using immunohistochemistry against the cell cycle protein Ki67 at 3 dpi in Aldh1l1eGFP reporter mice. Although many $\mathrm{Ki}^{+}{ }^{+}$cells were observed in adult neurogenic zones, only $1 / 3206 \mathrm{GFP}^{+}$astrocytes $(n=3$ animals) double-labeled for Ki67 and this cell was GFAP ${ }^{-}$in the cortical gray matter. In 3xSham control animals, 2/3618 $\mathrm{GFP}^{+}$ astrocytes ( $n=4$ animals) colabeled for Ki67 and colabeled cells were also GFAP ${ }^{-}$.

To ensure that a potentially small population of astrocytes reentering the cell cycle and that the proper time frame for capturing proliferating astrocytes was not missed, we next labeled all cells undergoing DNA synthesis with the DNA base analog BrdU. Aldh1l1-eGFP reporter mice were injected with BrdU twice daily starting at the day of single or rdTBI for 7 consecutive days. The number of $\mathrm{GFP}^{+} / \mathrm{BrdU}^{+}$cells was determined in the cortical gray matter of Sham and injured animals in areas where $\mathrm{GFAP}^{+}$ cells were present. In Sham mice, $0.1 \pm 0.1 \%$ of astrocytes had incorporated BrdU (1/946 $\mathrm{GFP}^{+}$astrocytes, $n=3$ animals). In mice with TBI, $0.6 \pm 0.2 \%$ (single TBI: $4 / 796 \mathrm{GFP}^{+}$astrocytes, $n=3$ animals) and $0.8 \pm 0.5 \%$ (rdTBI: $5 / 659 \mathrm{GFP}^{+}$astrocytes, $n=3$ animals) of astrocytes also labeled for BrdU (3xSham vs 1xTBI; $p=0.3448$, Kruskal-Wallis ANOVA with Dunn's multiple-comparisons test). These astrocytes accounted for $1.6 \pm$ $1.6 \%$ of the total $\mathrm{BrdU}^{+}$cell population in Sham mice, $5.2 \pm$ $1.0 \%$ and $7.0 \pm 3.5 \%$ in single and rdTBI animals, respectively. The remainder of the $\mathrm{BrdU}^{+}$population can likely be accounted for by slowly cycling NG2 cells and turnover of microglia (Simon et al., 2011).

We conclude from these data that rdTBI did not induce proliferation of the $\mathrm{GFAP}^{+}$astrocyte population.

\section{rdTBI induced an atypical astrocyte response}

To further assess the response of astrocytes to rdTBI, immunohistochemistry for key astrocyte proteins mediating homeostatic functions was performed. We found that the cortical gray matter of mice that underwent rdTBI contained areas devoid of astrocyte markers, including Kir4.1, Glt1, Connexin43 (Cx43), glutamine synthetase (GS), and $S 100 \beta$ compared with the consistent staining pattern in brain tissue from Sham animals (Fig. 3a; Fig. 3-1, available as Extended Data). When brain slices were stained against several astrocytic proteins simultaneously, for example, Kir4.1 and Glt1 together (Fig. 3b; Fig. 3-1, available as Extended Data), we observed an overlap between the areas with loss or reduction of expression, indicating that the same astrocytes were affected. Unbiased stereology revealed that $\sim 6 \%$ of the entire cortical area was affected by Glt1 loss, both at $1 \mathrm{dpi}(p=0.0104)$ and $14 \mathrm{dpi}(p=0.0066)$ compared with Sham controls (Fig. $3 c$, two-way ANOVA for variable "injury" TBI/Sham; for detailed statistics, see Fig. 3-1, available as Extended Data) and rdTBI males and females did not significantly differ (Fig. $3 d, p=0.8742$, two-way ANOVA for variable "sex"; for detailed statistics, see Fig. 3-1, available as Extended Data).

The astrocytic protein loss occurred either in single astrocytes scattered across the cortical gray matter and hippocampus or in larger groups of cells (Figs. 3, 4). This phenomenon was noted as early as $4 \mathrm{~h}$ after the first injury and persisted for at least 2 months (Fig. 4, Table 3).

We next assessed the expression pattern of the Aldh111-eGFP reporter, in which GFP expression is dependent on the activity of the astrocyte-specific Aldh1l1 promoter. In our experience, this reporter is upregulated in reactive astrocytes surrounding stab wounds or gliomas. After rdTBI, we found that the reduced GFP 


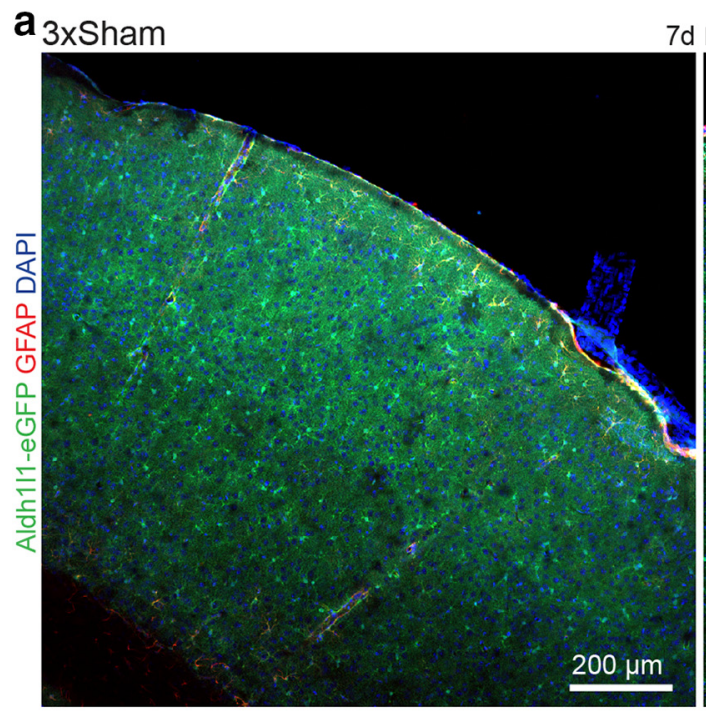

7d rdTBI

$7 \mathrm{dpi}$
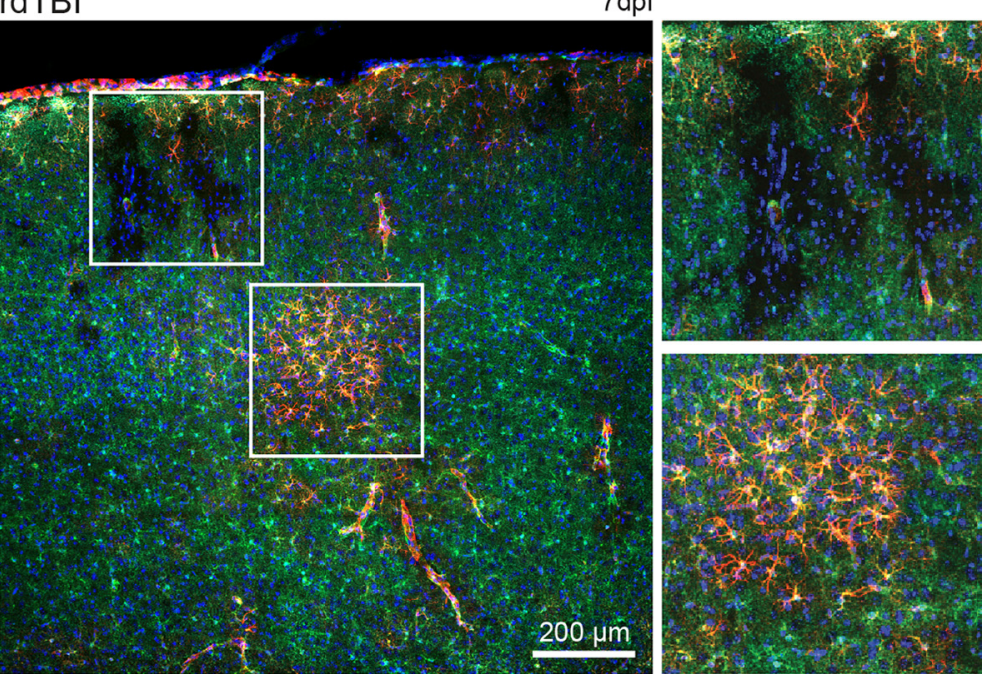

b 3xSham

$7 d$

rdTBI

$7 \mathrm{dpi}$
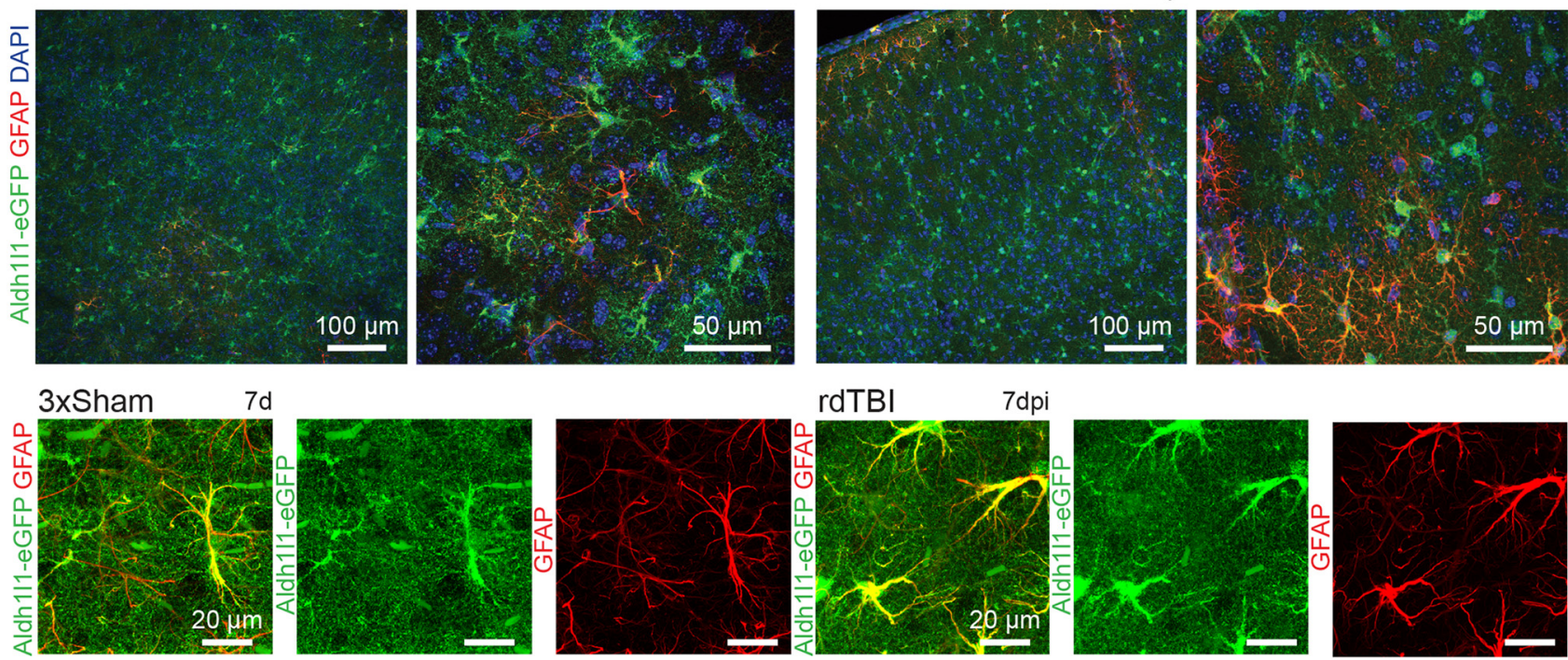

rdTBI

$7 \mathrm{dpi}$
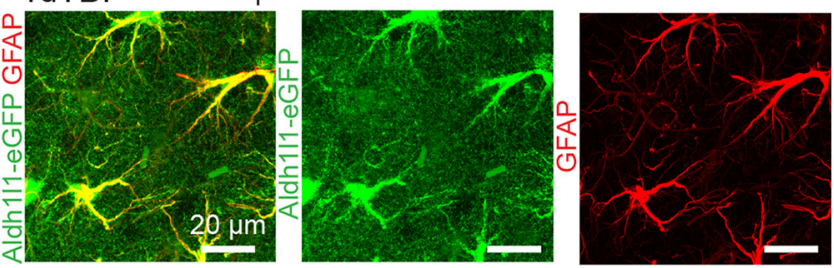

C Cortical gray matter Females

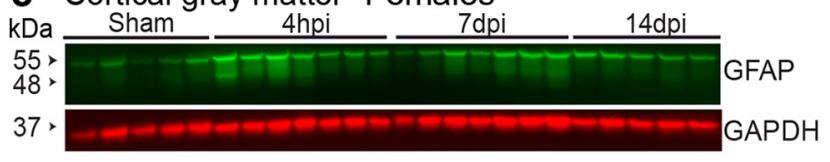

h Hippocampus Males

\section{d Females}

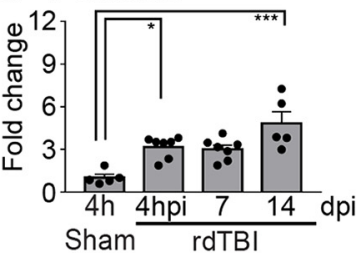

f Females(F)/Males(M)

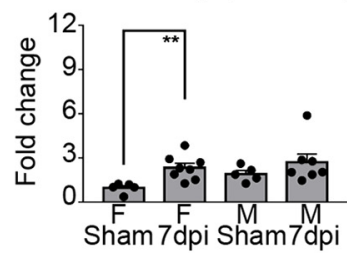

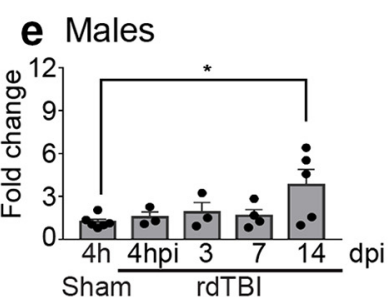

g Males- 7dpi expanded

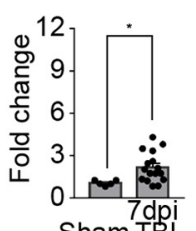

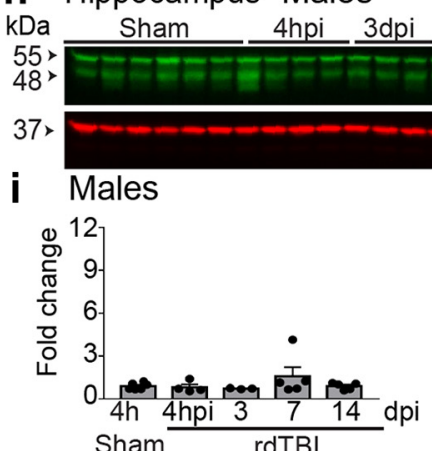

k Females(F)/Males(M)

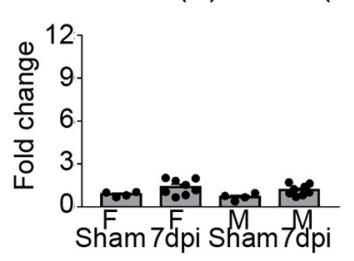

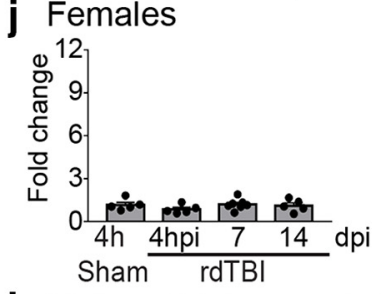

I Males - 7dpi expanded

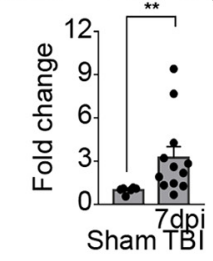

Figure 2. $\quad$ rdTBI caused mild upregulation of GFAP and hypertrophy in a subset of astrocytes. $\boldsymbol{a}$, rdTBI resulted in small groups of astrocytes scattered across the cortical gray matter that expressed GFAP at 7 dpi. Gray matter astrocytes in the forebrains of uninjured adult mice tile, resulting in an even distribution of Aldh111-eGFP reporter-positive astrocytes ( $3 \times$ Sham, $7 \mathrm{~d}$ ). After rdTBI, astrocytes disappeared or downregulated expression of the Aldh111-eGFP reporter (see also Fig. 4) indicated by areas with reduced levels of Aldh111-eGFP 7 dpi (rdTBI, and upper inset). (Figure legend continues.) 
a
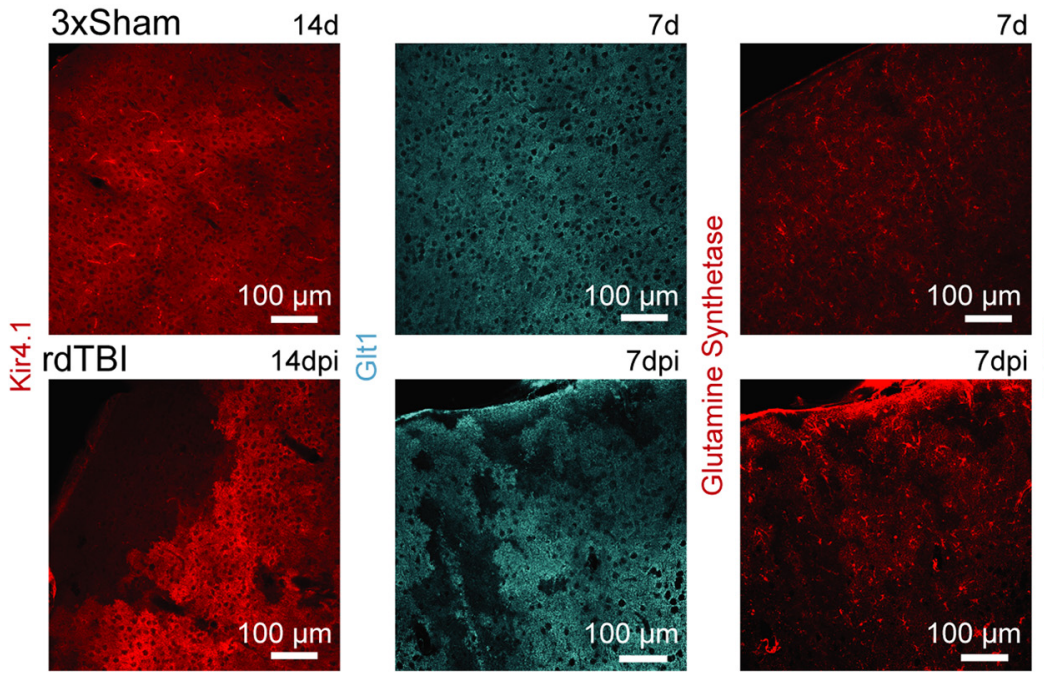

$\frac{8}{\cos }$
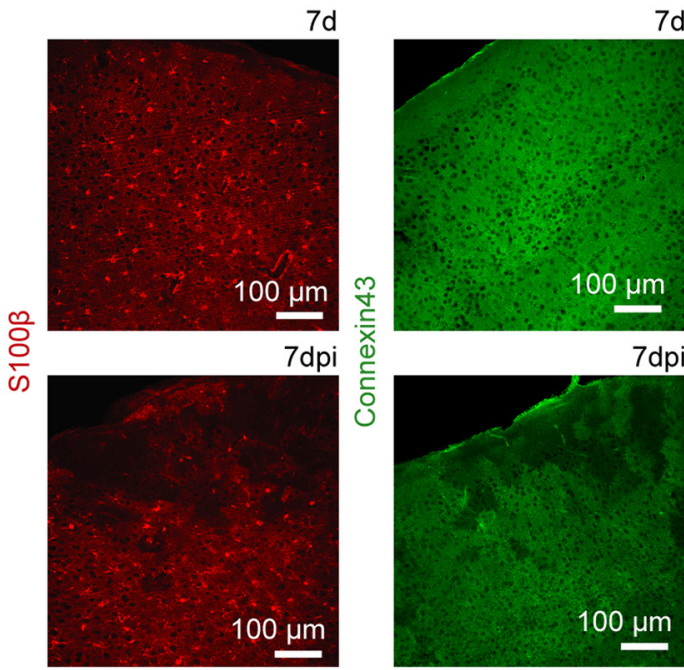

$7 d$

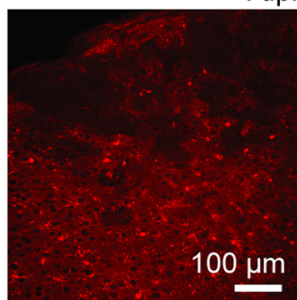

$7 \mathrm{dpi}$ b

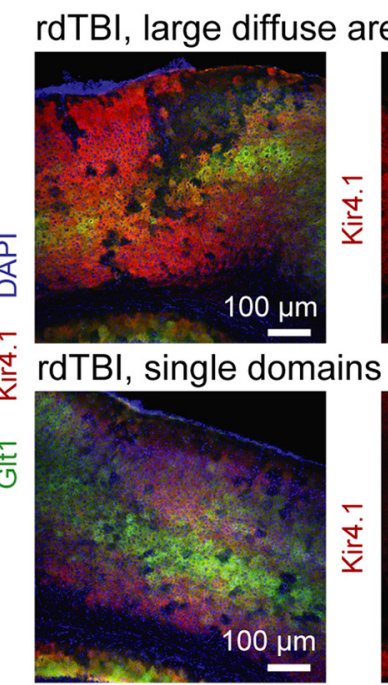

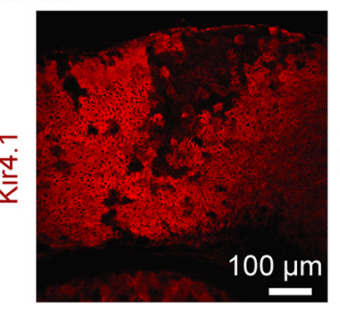

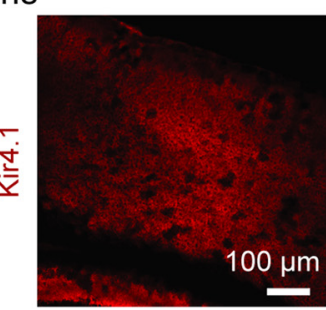

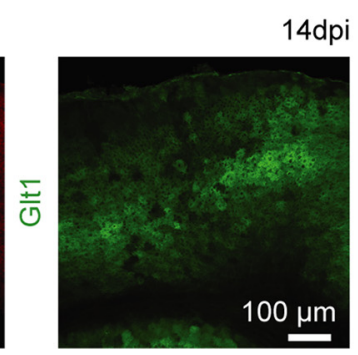

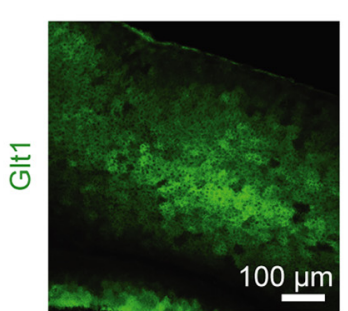

C

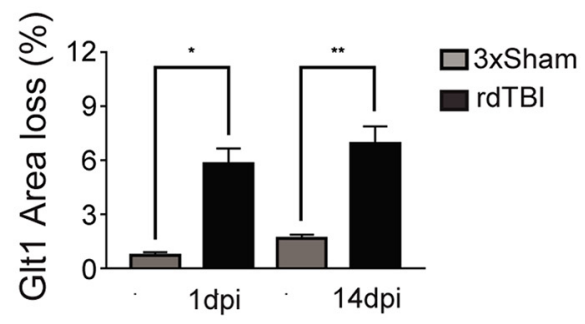

d

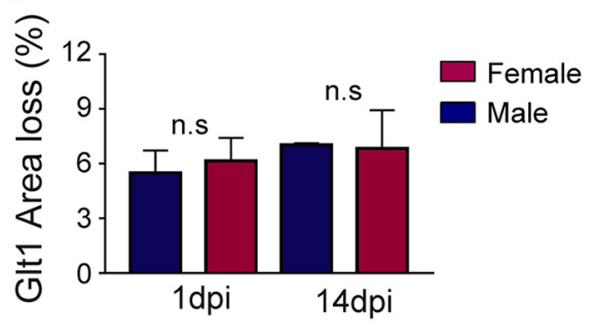

Figure 3. rdTBI induced atypical astrocytes. $\boldsymbol{a}$, Confocal imaging showed that the astrocyte proteins Kir4.1, GIt1, GS, S100 $\beta$, and Cx43 were downregulated in some astrocytes after rdTBI. GS and S100 $\beta$ were stained in the same brain slice. $\boldsymbol{b}$, rdTBI resulted in downregulation of Kir4.1 and GIt1 either in large areas or in single astrocyte domains scattered across the cortical gray matter at $14 \mathrm{dpi}$. Consistent DAPI density across these regions, seen in the images to the left, indicated intact tissue. $c$, Unbiased stereology quantified the total area without GIt1 within the cortical gray matter after rdTBI compared with 3xSham at $1 \mathrm{dpi}$ and $14 \mathrm{dpi}$. $\boldsymbol{d}$, Stratifying these data based on sex does not reveal significant differences. See Figure 3-1 (available as Extended Data) for breakdown of sample sizes dependent on sex for representative images and detailed numerical data for all statistical group comparisons. ${ }^{*} p \leq 0.05$, ${ }^{* *} p \leq 0.01$.

levels in peripheral fine processes of Aldh111-eGFP ${ }^{+}$astrocytes closely followed the loss of Glt1. Reduced GFP levels in Aldh111eGFP $^{+}$astrocytes occurred as early as $4 \mathrm{hpi}$ and was sustained for at least 2 months after rdTBI (Fig. $4 a$, Table 3). We did

$$
\leftarrow
$$

(Figure legend continued.) $\quad \boldsymbol{b}$, Astrocytes with upregulated GFAP showed swelling of processes compared with GFAP positive astrocyte in 3xSham at $7 \mathrm{dpi}$. c-I, GFAP levels were mildly increased after rdTBI. Western blot for GFAP in cortical gray matter of female mice at different time points after rdTBI ( $\boldsymbol{c}$; quantified in $\boldsymbol{d}$ for females and in $\boldsymbol{e}$ for males) compared male $(\boldsymbol{f})$ and female ( $\boldsymbol{g}$ ) mice and increased male sample number. Western blot for GFAP in hippocampus ofmale mice at different time points after rdTBI ( $\boldsymbol{h}$; quantified in $\boldsymbol{i}$ for males and in $\boldsymbol{j}$ for females) compared male $(\boldsymbol{k})$ and female $(\boldsymbol{l})$ mice with increased male sample number. See Figure 2-1 (available as Extended Data) for breakdown of sample sizes dependent on sex for representative images and detailed numerical data for all statistical group comparisons. ${ }^{*} p \leq 0.05,{ }^{* *} p \leq$ $0.01,{ }^{* * *} p \leq 0.001$. observe remnant Aldh1l1-eGFP ${ }^{+}$astrocyte cell bodies in the areas that lacked $\mathrm{GFP}^{+}$and Glt1 expression in peripheral processes. However, the number of $\mathrm{GFP}^{+}$astrocyte cell bodies was reduced by about half at $4 \mathrm{hpi}(p=0.0049), 3 \mathrm{dpi}$, and 28 dpi $(p<0.0001)$ (Fig. $4 b$, two-way ANOVA for variable "astrocyte density by location"; for detailed statistics, see Fig. 4-1, available as Extended Data). We next performed two-photon in vivo imaging through a thinned-skull cranial window, which enabled observing the same animal before and repeatedly after rdTBI. Histology suggested that areas with reduction of Aldh1l1-eGFP expression overlap with areas that also show a reduction in Glt1, Kir4.1, Cx43, S100 $\beta$, and GS. We thus used Aldh1l1-eGFP animals to identify astrocytes in vivo. First, an imaging protocol was developed that assured that exposure to the two-photon laser did not induce phototoxicity 
a
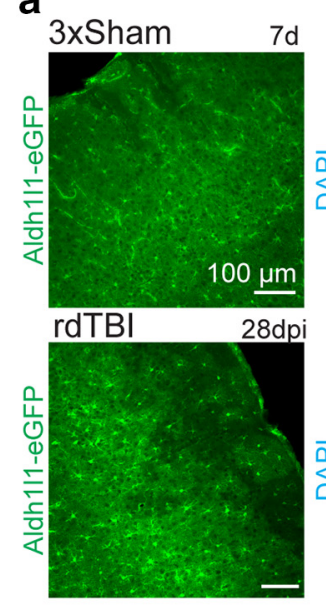
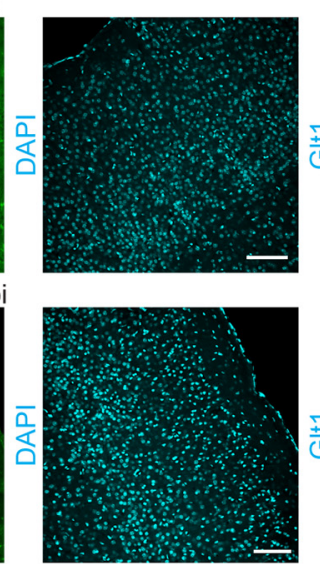
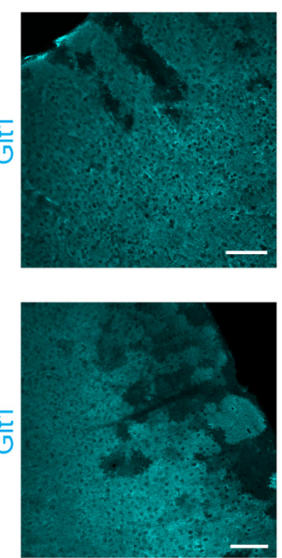

b
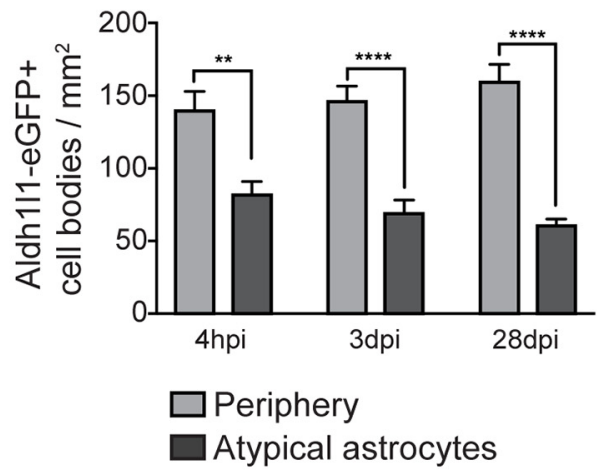

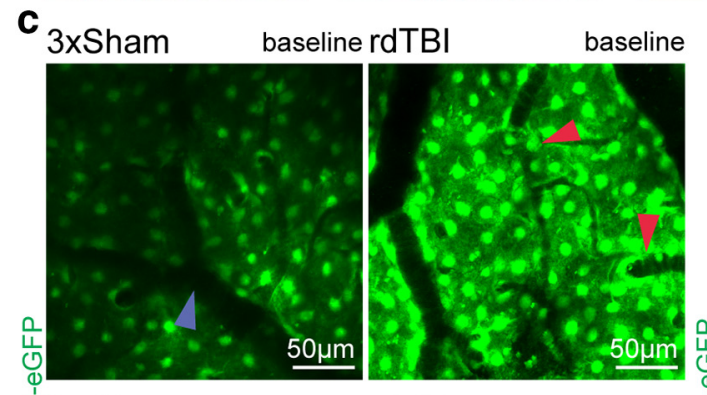

rdTBI baseline

1dpi

2dpi
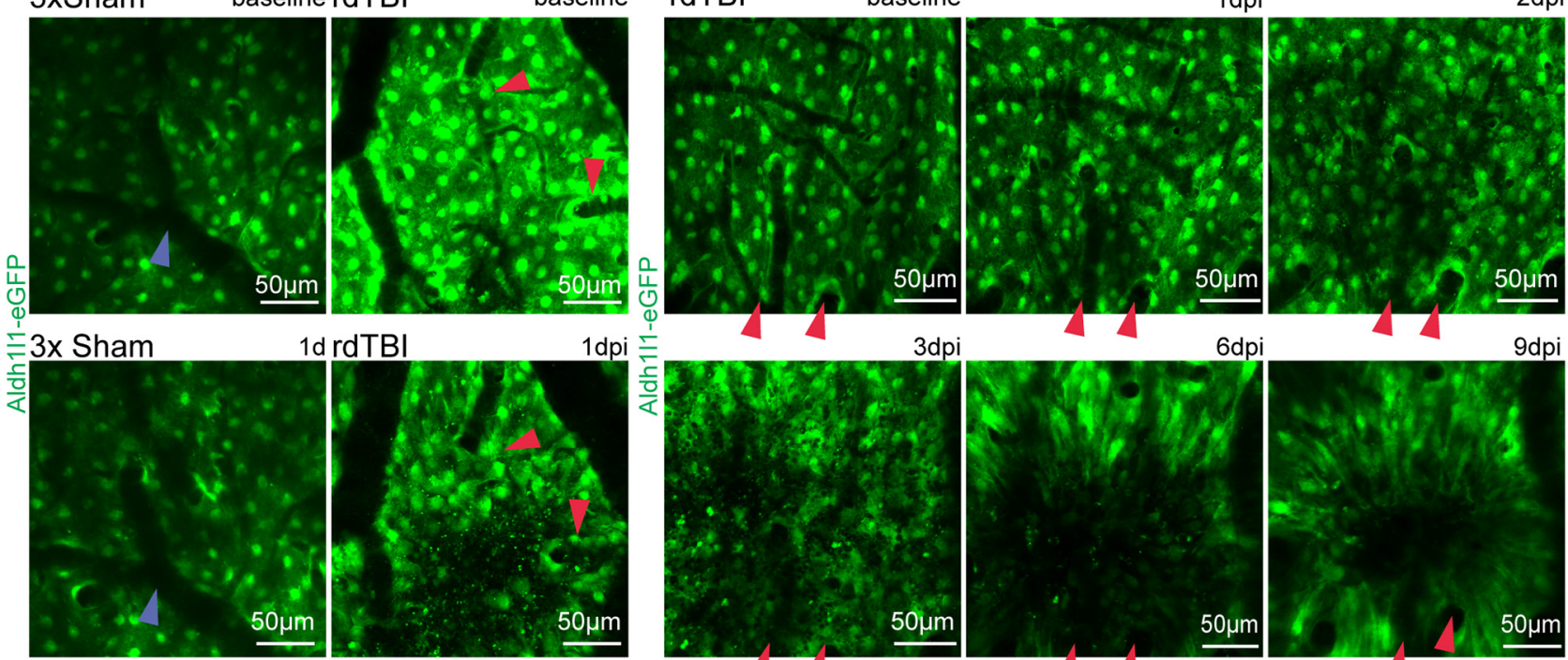

d rdTB।

$1 \mathrm{dpi} \stackrel{亡}{=}$

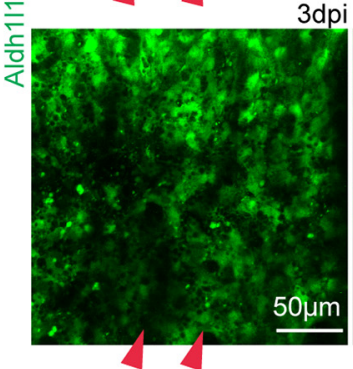

6dpi

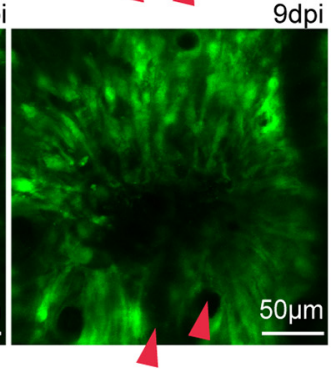

d
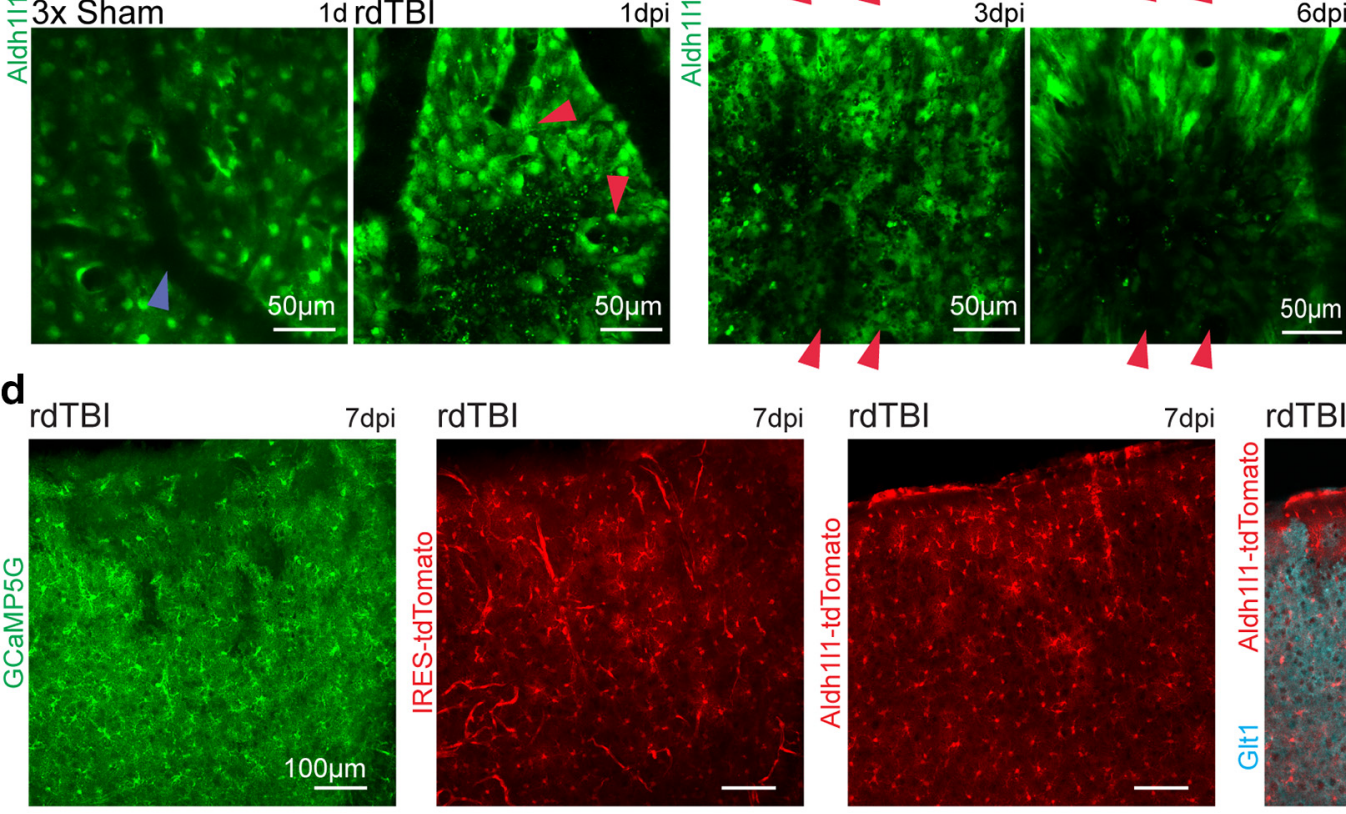

rdTBI

7dpi rdTBI

$7 \mathrm{dpi}$
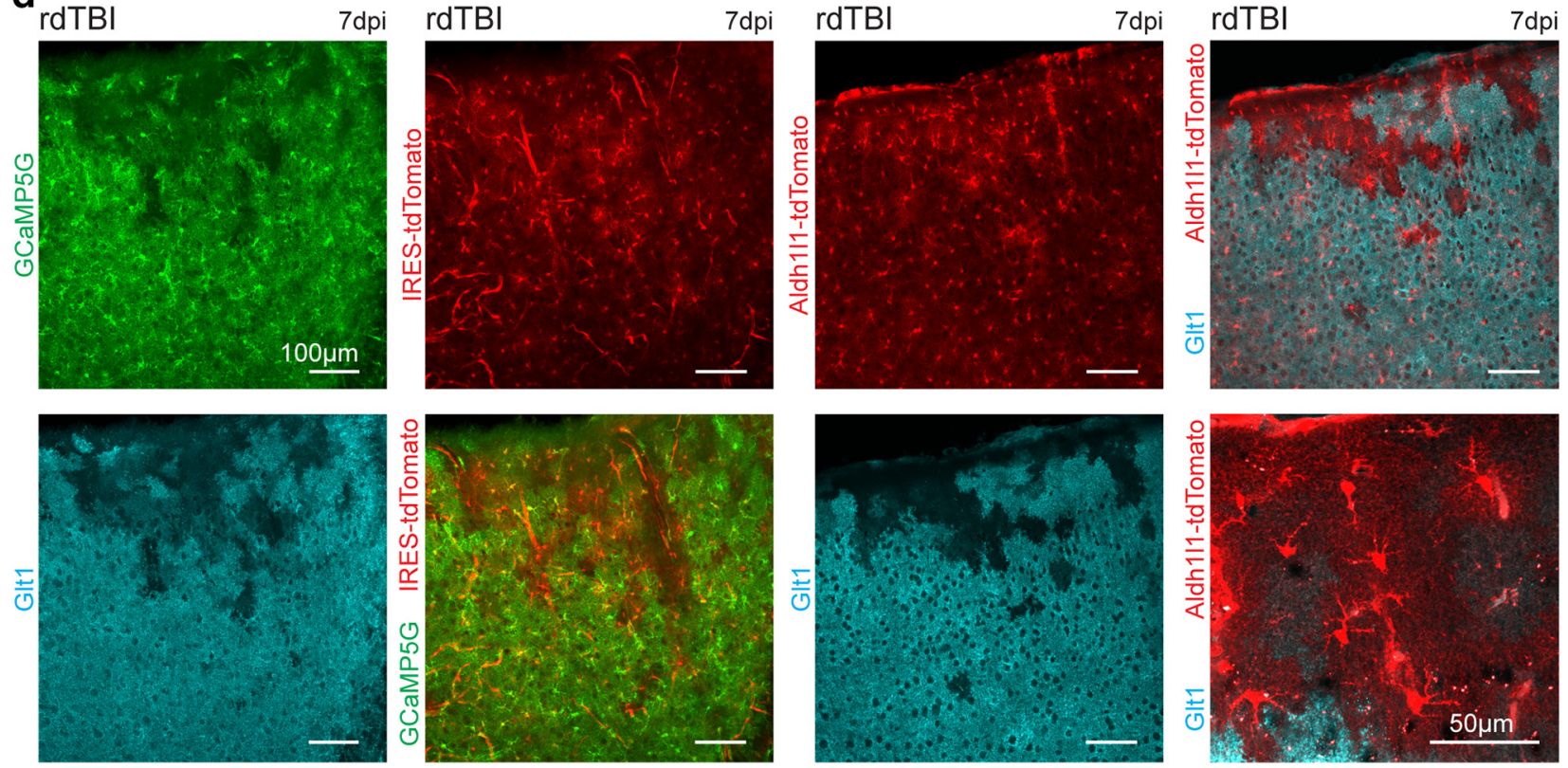

Figure 4. Repetitive mild/concussive TBI caused early and sustained astrocyte protein downregulation. $\boldsymbol{a}$, Confocal imaging at several time points showed consistent downregulation of the Aldh111-eGFP reporter GIt1 and GCaMP5G in the cortical gray matter after rdTBI. DAPI staining indicated tissue integrity throughout these regions. $\boldsymbol{b}$, Quantification of Aldh111-eGFP ${ }^{+}$cell bodies in areas with Aldh111-eGFP ${ }^{+} /$Glt1 loss astrocytes compared with the peripheral regions surrounding these areas. c, Repeated two-photon imaging in the same Aldh111-eGFP animal before and after rdTBI. Aldh111-eGFP loss was observed starting 1 dpi and was at its maximum on day 9 after rdTBI. Blue (sham) and red (rdTBI) arrows indicate stable "landmarks" (Figure legend continues.) 
Table 3. Summary of protein expression changes observed in immunohistochemistry of atypical astrocytes

\begin{tabular}{|c|c|c|c|c|c|c|c|c|}
\hline \multirow[b]{2}{*}{ Astrocyte marker } & \multicolumn{8}{|c|}{ Time point } \\
\hline & Acute & $4 \mathrm{hpi}$ & $1 \mathrm{dpi}$ & $3 \mathrm{dpi}$ & $7 \mathrm{dpi}$ & $14 \mathrm{dpi}$ & $28 \mathrm{dpi}$ & $56 \mathrm{dpi}$ \\
\hline GFAP & $\mathrm{N} / \mathrm{A}$ & $\mathrm{N} / \mathrm{A}$ & $\downarrow$ & $\mathrm{N} / \mathrm{A}$ & $\downarrow$ & $\downarrow$ & $\mathrm{N} / \mathrm{A}$ & N/A \\
\hline Kir 4.1 & N/A & $\downarrow$ & $\mathrm{N} / \mathrm{A}$ & $\mathrm{N} / \mathrm{A}$ & $\downarrow$ & $\downarrow$ & N/A & N/A \\
\hline Glt1 & $\downarrow$ & $\downarrow$ & $\downarrow$ & $\downarrow$ & $\downarrow$ & $\downarrow$ & $\downarrow$ & $\downarrow$ \\
\hline GS & $\mathrm{N} / \mathrm{A}$ & $\downarrow$ & $\mathrm{N} / \mathrm{A}$ & N/A & $\downarrow$ & $\mathrm{N} / \mathrm{A}$ & $\mathrm{N} / \mathrm{A}$ & $\mathrm{N} / \mathrm{A}$ \\
\hline$S 100 \beta$ & $\downarrow$ & $\downarrow$ & $\downarrow$ & $\downarrow$ & $\downarrow$ & $\downarrow$ & $\downarrow$ & $\downarrow$ \\
\hline CX43 & $\mathrm{N} / \mathrm{A}$ & $\mathrm{N} / \mathrm{A}$ & $\mathrm{N} / \mathrm{A}$ & $\mathrm{N} / \mathrm{A}$ & $\downarrow$ & $\mathrm{N} / \mathrm{A}$ & $\mathrm{N} / \mathrm{A}$ & N/A \\
\hline Aldh1l1 eGFP & $\downarrow$ & $\downarrow$ & $\downarrow$ & $\downarrow$ & $\downarrow$ & $\downarrow$ & $\downarrow$ & $\downarrow$ \\
\hline GCaMP5G & $\downarrow$ & N/A & $\downarrow$ & $\mathrm{N} / \mathrm{A}$ & $\downarrow$ & $\mathrm{N} / \mathrm{A}$ & $\mathrm{N} / \mathrm{A}$ & $\mathrm{N} / \mathrm{A}$ \\
\hline IRES tdTomato & $\uparrow$ & $\uparrow$ & $\uparrow$ & N/A & $\uparrow$ & $\mathrm{N} / \mathrm{A}$ & $\mathrm{N} / \mathrm{A}$ & $\mathrm{N} / \mathrm{A}$ \\
\hline Aldh1l1 tdTomato & $\mathrm{N} / \mathrm{A}$ & $\mathrm{N} / \mathrm{A}$ & N/A & $\mathrm{N} / \mathrm{A}$ & $\uparrow$ & $\mathrm{N} / \mathrm{A}$ & $\mathrm{N} / \mathrm{A}$ & $\mathrm{N} / \mathrm{A}$ \\
\hline
\end{tabular}

Note that whereas GFAP was absent in atypical astrocytes, it was upregulated in other subsets of astrocytes after rdTBI.

N/A, Not applicable.

and loss of GFP expression in uninjured Aldh111-eGFP mice. Baseline imaging (before rdTBI) was performed after the thinned-skull window was prepared. Unfortunately, although astrocyte cell bodies were readily visible in Aldh1l1-eGFP mice, finer processes could not be resolved to study subtle changes in astrocyte morphology after rdTBI. After baseline images were obtained, mice underwent rdTBI followed by post-TBI imaging (see detailed step-by-step protocol in Shandra and Robel, 2019). We did observe loss of reporter expression in two of four rdTBI mice, but no changes were observed in the other two rdTBI animals or Sham controls (Fig. $4 c, d$; Fig. 4-1, available as Extended Data). Given that the imaging window is relatively small, the imaging depth is limited to $\sim 100 \mu \mathrm{m}$, and the location of "damage" resulting from rdTBI is unpredictable, it is possible that areas with reporter loss were missed in some rdTBI mice. In summary, two-photon in vivo imaging confirmed the reduction in Aldh1l1-eGFP reporter expression in response to rdTBI.

Finally, to determine whether astrocyte proteins and reporters were downregulated in classically reactive astrocytes, we used Aldh1l1-eGFP reporter mice and colabeled with an antibody against the astrogliosis marker GFAP. Importantly, not only were astrocytes in these areas negative for GFAP, we also did not observe upregulation of GFAP in astro-cytes surrounding these areas (Fig. 2a, box; Fig. 2-1, available as Extended Data).

\section{Atypical astrocytes do not undergo cell death after rdTBI}

To determine whether astrocytes might be dying, we attempted to colabel the remaining Aldh1l1-eGFP ${ }^{+}$cell bodies in areas with reduced Glt1/Aldh1l1-eGFP reporter staining with immunohistochemistry against the apoptosis marker cleaved Caspase-3 or used fluorescent Terminal deoxynucleotidyl transferase dUTP Nick-End Labeling (TUNEL). Although controls determined that the assays worked, astrocytes did not label with TUNEL or cleaved caspase- 3 acutely ( $4 \mathrm{hpi}$ ) or at later time points ( $28-56$ dpi) after rdTBI.

\section{$\leftarrow$}

(Figure legend continued.) to aid orientation within the images. $\boldsymbol{d}$, Astrocytes are presented in areas with GIt1 and GCaMP5G downregulation as indicated by the presence of other two astrocyte-specific reporters expressing the fluorescent protein tdTomato. See Figure 4-1 (available as Extended Data) for breakdown of sample sizes dependent on sex for representative images and detailed numerical data for all statistical group comparisons. ${ }^{* *} p \leq 0.01,{ }^{* * * *} p \leq$ 0.0001 .
These data were further corroborated by quantifying cell densities by counting DAPI ${ }^{+}$nuclei in areas with Aldh1l1-eGFP reporter loss compared with surrounding cortical areas. Peripheral areas, rather than Sham animals, were chosen as controls because cell densities vary significantly from cortical layer to cortical layer and along the anterior-posterior axis, making it difficult to exactly match brain regions in Sham animals to the regions with reporter loss in mice that underwent rdTBI. Cell densities were unchanged at $4 \mathrm{hpi}$ (area with reduced of reporter expression: $2128 \mathrm{DAPI}^{+}$nuclei $/ \mathrm{mm}^{2}$, periphery: $2110 \mathrm{DAPI}^{+}$nuclei $/ \mathrm{mm}^{2}$, $p=0.9994), 3 \mathrm{dpi}$ (area with lack of reporter expression: 1705 DAPI $^{+}$nuclei/ $/ \mathrm{mm}^{2}$, periphery: $1814 \mathrm{DAPI}^{+}$nuclei $/ \mathrm{mm}^{2}, p=$ 0.8792), and $28 \mathrm{dpi}$ (area with lack of reporter expression: 1693 $\mathrm{DAPI}^{+}$nuclei $/ \mathrm{mm}^{2}$, periphery: $2068 \mathrm{DAPI}^{+}$nuclei $/ \mathrm{mm}^{2}, p=$ 0.0825, two-way ANOVA with Sidak's multiple-comparisons test). Proliferation assays using immunohistochemistry against either Ki67 or BrdU at 3 dpi and $7 \mathrm{dpi}$, respectively, revealed that these astrocytes did not undergo cell division (Sham: 0/636 $\mathrm{GFP}^{+} / \mathrm{BrdU}^{+}$astrocytes; single $\mathrm{TBI}$ : $0 / 330 \mathrm{GFP}^{+} / \mathrm{BrdU}^{+}$astrocytes, rdTBI: $0 / 331$ astrocytes were $\mathrm{GFP}^{+} / \mathrm{BrdU}^{+} ; n=3,2$ females, 1 male in each group).

To determine whether only expression of astrocyte-specific proteins was affected, we took advantage of the genetically encoded GCaMP5G Calcium indicator (Gee et al., 2014), which was conditionally expressed in astrocytes after Cre-mediated excision of a stop cassette using the mouse GFAP-Cre line 73.12 (Herrmann et al., 2008) (C57BL/6 genetic background). Once Cre recombination took place during postnatal development, GCaMP5G is continuously transcribed from the strong synthetic promoter CAG and should thus not be affected by injury-induced changes to the astrocyte transcriptome. GCaMP5G is a GFP derivative and can be detected with antibody staining against GFP. However, GCaMP5G was also downregulated in the Glt $1^{-}$subsets of parenchymal astrocytes (Fig. 4a; Fig. 4-1, available as Extended Data).

We used a third reporter that expressed the red fluorescent protein tdTomato as part of the GCaMP5G reporter (C57BL/6 genetic background), but is translated from an internal ribosomal entry site (IRES) rather than the $5^{\prime}$-untranslated region. Surprisingly, expression of this reporter is enhanced in astrocytes lacking Glt1 or GCamp5G expression at 7 dpi (Fig. 4a; Fig. 4-1, available as Extended Data). We speculated that IRES-mediated translation was enhanced in response to rdTBI as IRES-mediated proteins can be upregulated as part of the cell stress response (Komar and Hatzoglou, 2011). However, the Aldh1l1-tdTomato reporter mice (FVB/N//Crl:CD1(ICR) mixed background), in which tdTomato expression is dependent on Aldh1l1 as a promoter and is not regulated by an IRES, responded similarly to the IRES-tdTomato reporter with an increase in tdTomato expression in areas of Glt1 downregulation at 7 dpi (Fig. 4a; Fig. 4-1, available as Extended Data). The genetic background did not appear to be the factor determining whether a reporter was upregulated or downregulated because either response was observed in transgenic strains on $\mathrm{C} 57 \mathrm{BL} / 6$ and $\mathrm{FVB} / \mathrm{N}$ background. Aldh1l1-reporter mice are both BAC transgenic lines created by the GENSAT project and the integration site(s) of the BAC constructs are unknown. It is thus possible that differing genomic regions surrounding the transgene caused the contradictory responses of both Aldh1l1-reporters to rdTBI. Alternatively, the raw fluorescence of the GFP, GCAMP5G, and tdTomato fluorophores may be affected differentially by potential injury-induced changes of the intracellular $\mathrm{pH}$ of astrocytes. 
Atypical astrocytes are uncoupled from the astrocytic network

Next, we determined whether normal astrocyte functions were impaired in atypical astrocytes. Cx43 is the main gap junctional protein in astrocytes crucial for their ability to form a functional syncytium (Dermietzel et al., 1991; Griemsmann et al., 2015; Bouchat et al., 2018). The integrity of this network is essential to astrocytic function and loss of gap junctional coupling has been associated with epilepsy (Steinhäuser et al., 2012; Pannasch and Rouach, 2013; Bedner et al., 2015). To test whether atypical astrocytes were uncoupled due to their lack of $\mathrm{Cx} 43$ (and possibly other uninvestigated gap junctional proteins), we patched on Aldh1l1-tdTomato ${ }^{+}$astrocytes in acute brain slices 7-15 dpi. The internal solution contained biocytin, a small molecule able to pass through gap junctions. In healthy astrocyte networks, biocytin diffused from the originally patched cells to neighboring astrocytes labeling cells even far from the "cell of origin" (Konietzko and Müller, 1994; Condamine et al., 2018). Of 43 slices (13 animals) with biocytin-filled astrocytes, 18 contained atypical astrocytes characterized by the presence of Aldh1l1-tdTomato but absence of Glt1. The area of astrocytes with biocytin-labeling was quantified in atypical tdTomato ${ }^{+} / \mathrm{Glt}^{-}$and neighboring tdTomato ${ }^{+} / \mathrm{Glt}{ }^{+}$areas (Fig. 5). Biocytin was preferentially excluded from atypical astrocytes (Fig. $5 c, d$ ) (paired $t$ test, $p<0.0001$ ), suggesting disrupted coupling between atypical astrocytes and their neighbors.

\section{rdTBI causes mild activation of microglia}

We next determined whether microglia responded to rdTBI and if their response differed in regions with atypical astrocytes. Immunohistochemistry against the pan-microglia marker Ibal and Cd11b, which is upregulated in reactive microglia, was performed in Sham and rdTBI Aldh1l1-eGFP mice 4 hpi, 1 dpi, 3 dpi, 7 dpi, and 56 dpi. Confocal images were taken after rdTBI within regions with atypical astrocytes, in regions without atypical astrocytes (rdTBIr), and in matching brain slices from Sham animals. A pronounced injuryinduced increase in $\mathrm{Cd} 11 \mathrm{~b}$ labeling was absent when comparing ROIs between Sham, rdTBI and rdTBIr (Fig. $6 a$; for breakdown of group sizes, see Fig. 6-1, available as Extended Data). We did note an increase in Ibal immunofluorescence intensity in rdTBI tissues. At $4 \mathrm{hpi}, 3 \mathrm{dpi}$, and 7 $\mathrm{dpi}$, this increase seemed to be restricted to areas with atypical astrocytes. At $1 \mathrm{dpi}$, areas without atypical astrocytes showed a similar increase in Iba1. At 56 dpi, Iba1 intensity was comparable among Sham, rdTBI, and rdTBIr (Fig. 6a; Fig. 6-1, available as Extended Data). Higher-resolution images revealed a thickening of microglia processes until $7 \mathrm{dpi}$, whereas this appeared to be resolved at $56 \mathrm{dpi}$ in areas with atypical astrocytes. Occasionally, microglia densities appeared reduced in parts of areas with atypical astrocytes (Fig. 6b). Total cortical Iba1 protein levels assessed by Western blot doubled at $7 \mathrm{dpi}$ $(n=15, p=0.0359)$ compared with Sham controls $(n=8$, Kruskal-Wallis ANOVA with Dunn's multiple comparisons test; data from male and female blots were pooled for analysis; Fig. $6 c, d)$. Microglia appeared to respond mildly to rdTBI.

\section{Atypical astrocytes overlap with areas of neuronal stress but} unchanged neuronal density after rdTBI

To determine whether atypical astrocytes influenced neuronal survival, we used the pan-neuronal marker NeuN or fluorescent Nissl staining and quantified neuronal densities within areas of atypical astrocytes compared with neighboring areas of cortical a
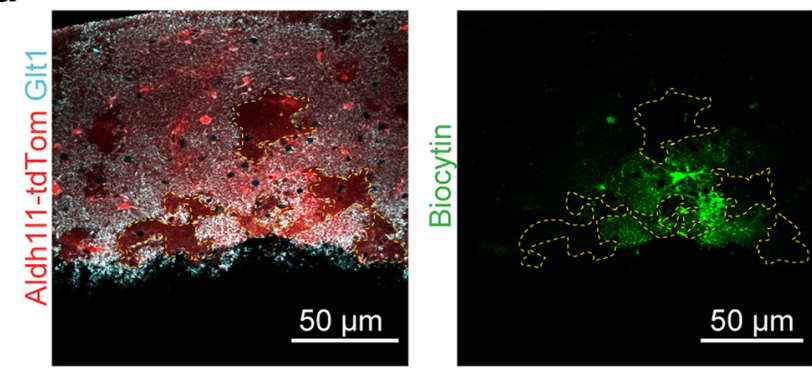

b

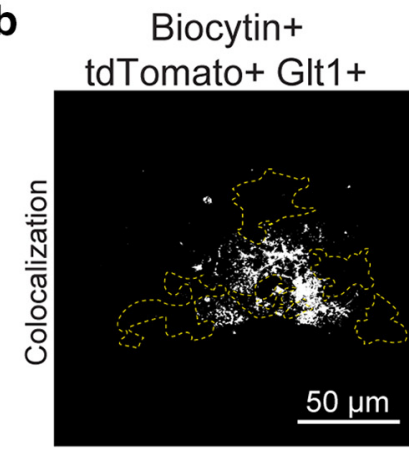

Biocytin+ tdTomato+ Glt1-

C
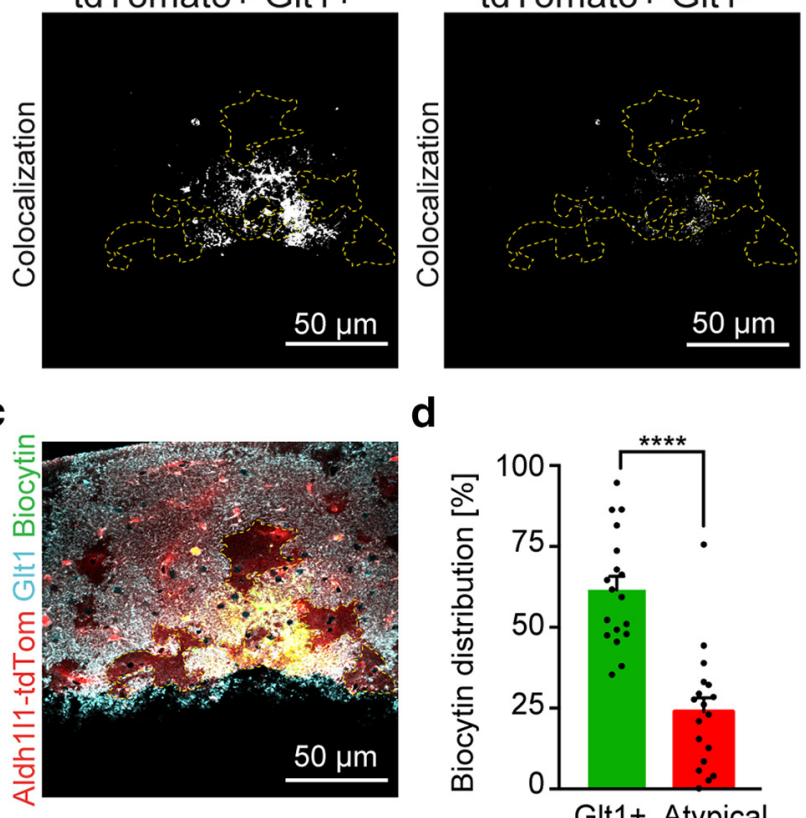

d

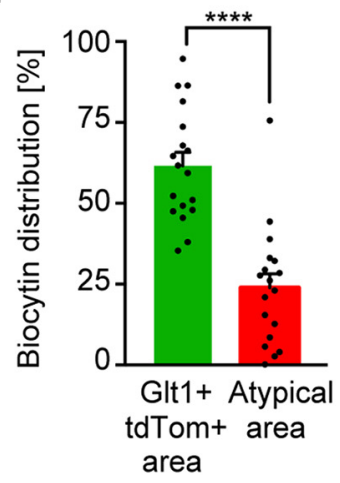

Figure 5. Biocytin is preferentially excluded of areas with atypical astrocytes. $\boldsymbol{a}$, Confocal image of an acute brain slice that was labeled with Glt1 Aldh1l1-tdTomato following biocytin filling of a single astrocyte using whole-cell patch clamping. Biocytin spreads to neighboring astrocytes through gap junctions. Atypical astrocytes were outlined by a yellow dotted line. $\boldsymbol{b}$, Binary image showing the biocytin-positive pixels that overlap with Glt- ${ }^{+}{ }^{+}$tdTomato ${ }^{+}$(left) and with the atypical astrocytes (right). c, Merged image of the tdTomato, Glt1, and biocytin channels. $\boldsymbol{d}$, Graph showing the distribution of biocytin between the $\mathrm{Glt}_{-1}{ }^{+}$tdTomato ${ }^{+}$area and the atypical area. If biocytin were evenly distributed between atypical areas and $\mathrm{TdTom}^{+} / \mathrm{Glt} 1{ }^{+}$area, then each area would have an expected value of $50 \%$ biocytin pixels and both areas add to a total value of $100 \%$. See Figure 5-1 (available as Extended Data) for breakdown of sample sizes dependent on sex for representative images and detailed numerical data for all statistical group comparisons. ${ }^{* * *} p \leq 0.0001$.

layer II/III. Layer I (included in the outline of areas with atypical astrocytes in Fig. 7) contains only few neurons and areas of atypical astrocytes rarely reached into cortical layers V and VI. Layer IV was excluded for quantification due to the pronounced difference in neuronal densities compared with layers II/III. Within areas of atypical astrocytes, downregulation or redistribution of $\mathrm{NeuN}$ was sometimes noted, especially when these areas were large (Fig. 7a; Fig. 7-1, available as Extended Data). NeuN downregulation or redistribution occurred as early as 1 dpi and persisted for at least $28 \mathrm{dpi}$. This phenomena has previously been described as a stress response of neurons to different kinds of injury (Robel et al., 2011a; Gusel'nikova and Korzhevskiy, 2015). 
a
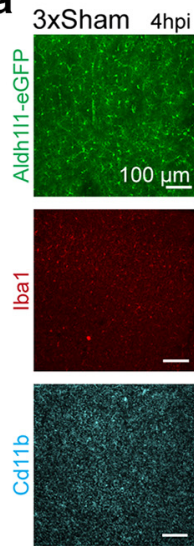

3xSham 56dpi rdTBI
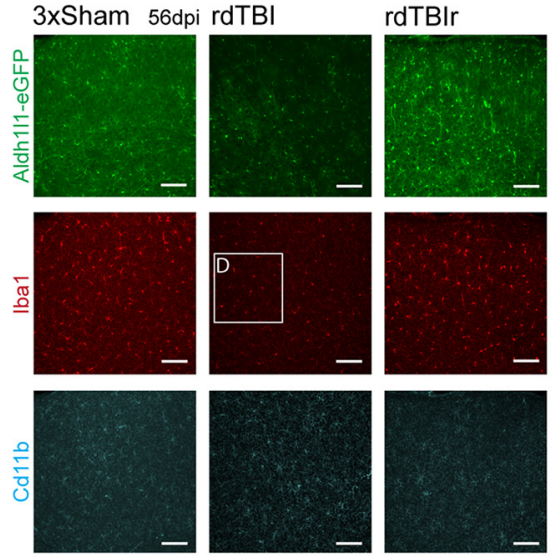

C Cortical gray matter

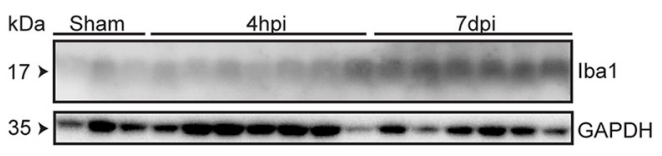

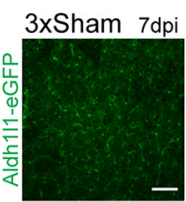
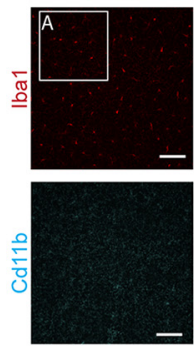

b 7 dpi $3 x$ Sham
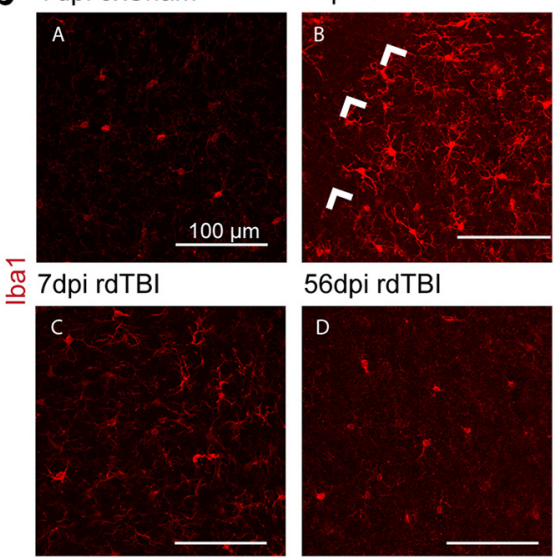

$56 d p i$ rdTBI

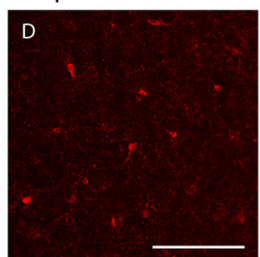

d Iba1 expression in cortical gray matter

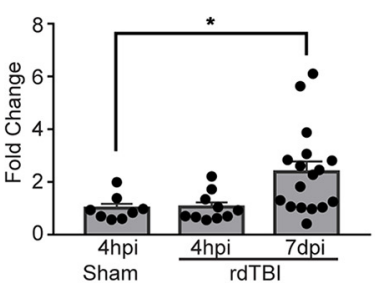

Figure 6. Microglia were mildly activated after rdTBI. $\boldsymbol{a}$, Confocal images within regions with atypical astrocytes (rdTBI), in regions without atypical astrocytes (rdTBIr), and in matching brain slices from Sham animals after staining for lba1 and Cd11b showed Iba1 upregulation at $4 \mathrm{hpi}$ and $7 \mathrm{dpi}$ and was resolved at $56 \mathrm{dpi}$. $\boldsymbol{b}$, Enlarged images with normal Iba 1

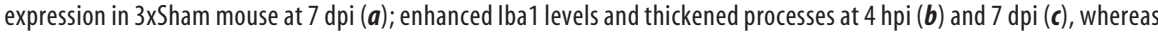
this was resolved at $56 \mathrm{dpi}$ in areas with atypical astrocytes (d). 0ccasionally, microglia densities appeared reduced in parts of areas with atypical astrocytes ( $\boldsymbol{b}$, arrows). $\boldsymbol{c}$, Western blots for lba1 compared expression in female (pictured) and male cortical gray matter across postinjury time points (quantified in $\boldsymbol{d}$ ). See Figure 6-1 (available as Extended Data) for breakdown of sample sizes dependent on sex for representative images and detailed numerical data for all statistical group comparisons. * $p \leq 0.05$.

To ensure that neuronal densities were accurately determined and that all neurons including NeuN-deficient ones were counted, fluorescent Nissl staining was used (Fig. 7b; Fig. 7-1, available as Extended Data). No difference in neuronal densities was observed within areas of astrocyte dysfunction up to $28 \mathrm{dpi}$ (Fig. $7 c, p=0.6998$ for variable "neuronal density by location," Kruskal-Wallis ANOVA with Dunn's multiple-comparisons test; Fig. 7-1, available as Extended Data), suggesting that the lack of expression of astrocyte homeostatic proteins did not immediately induce neuronal death. However, we cannot exclude functional changes in those neurons.
Repetitive diffuse TBI caused

spontaneous, unprovoked, recurrent seizures

TBI can induce epileptogenesis, a process during which neuronal networks are modified, ultimately resulting in network hyperexcitability or even unprovoked recurrent seizures. To determine whether global neuronal function was affected by rdTBI, we continuously monitored brain function using videoEEG. Given that seizure frequencies reported in rodent models of PTE are low (Pitkänen and Immonen, 2014), mice were fitted with EEG electrodes immediately following rdTBI and recorded continuously $24 / 7$ starting at $4 \mathrm{dpi}$ for up to $110 \mathrm{~d}$ after rdTBI.

Overall, 4 of 9 rdTBI animals presented with spontaneous recurrent seizures, whereas no seizures were observed in Sham mice $(n=6)$ and the remaining 5 rdTBI animals (Fig. 8a-d). Three of these 4 animals had convulsive seizures characterized by freezing, facial automatisms (Racine stage 1), head nodding and tail extension (Racine stage 2), and sometimes progression to bilateral forelimb clonus, rearing and falling (Racine stage 5) (Fig. 8c). One animal experienced a seizure cluster (five seizures on the same day, with return to baseline EEG between the ictal events). Before and/or after the ictal episode intermittent epileptiform interictal activity occurred, represented by paroxysmal spike discharges (defined as sharp-shaped potentials of 30-70 ms and spike-sharp wave discharges of $<200 \mathrm{~ms}$ ). A postictal EEG background depression was observed after each stage 5 convulsive seizure (Fig. 8c). One rdTBI mouse presented with recurrent episodes of nonconvulsive seizures with uniform morphology, unilateral or bilateral origin spike-wave discharges emerging during sleep and/or wakefulness. During wakefulness, nonconvulsive seizures were accompanied by "freezing behavior" or behavioral arrest lasting on average 20-30 s (Fig. $8 d$ ). One rdTBI mouse presented with only one nonconvulsive seizure. Occurrence of two spontaneous seizures spaced apart by at least $24 \mathrm{~h}$ is used as minimum criterion for an epilepsy diagnosis in humans. We thus excluded this mouse from quantifications of seizure incidence, frequency, power spectrum analysis, and Glt1 area loss fraction quantification.

Power spectral analysis showed that convulsive seizures consisted predominantly of $30-40 \mathrm{~Hz}$ frequency bands (gamma rhythm) at seizure onset, with postictal depression at $4.5-8.5 \mathrm{~Hz}$ frequency (theta rhythm) (Fig. 8e). Nonconvulsive seizures had frequency power density spectra similar to animals with convulsive seizures 
$a_{\text {rdTBI }}$

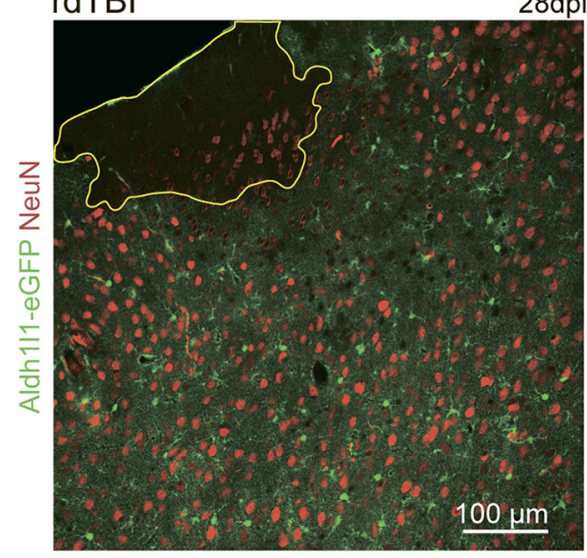

28dpi

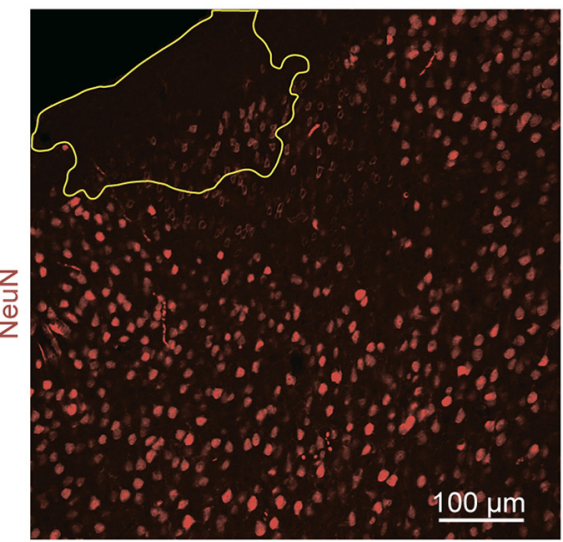

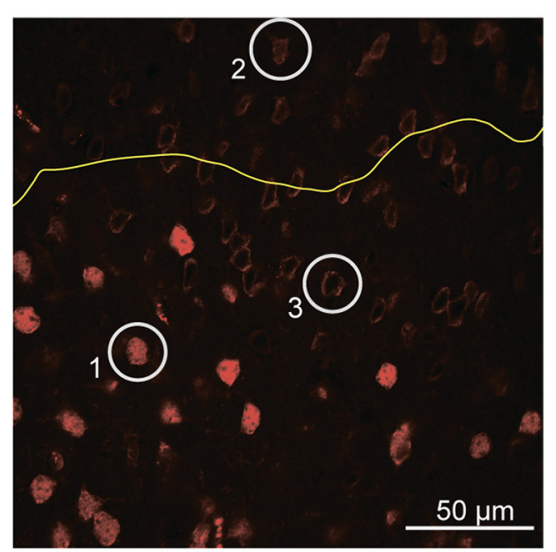

$\mathbf{b}_{\text {rdTBI }}$
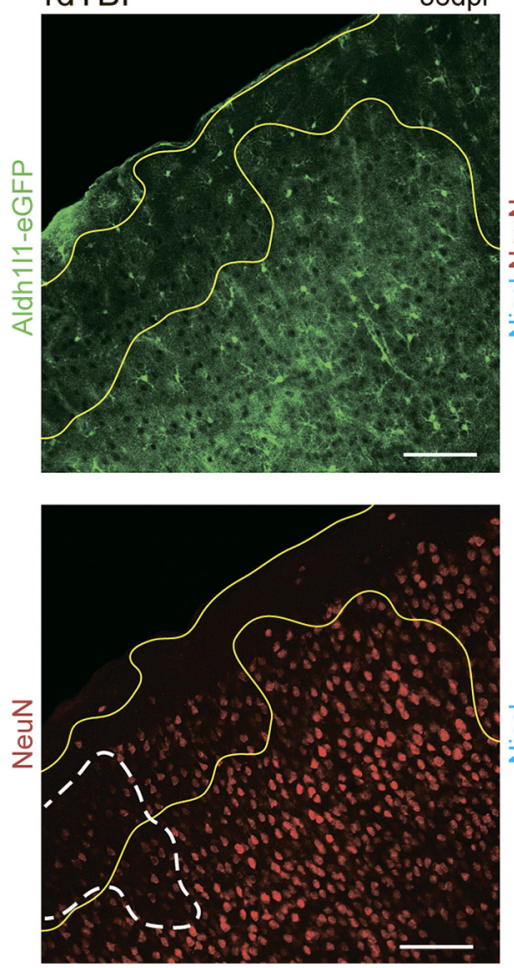

C
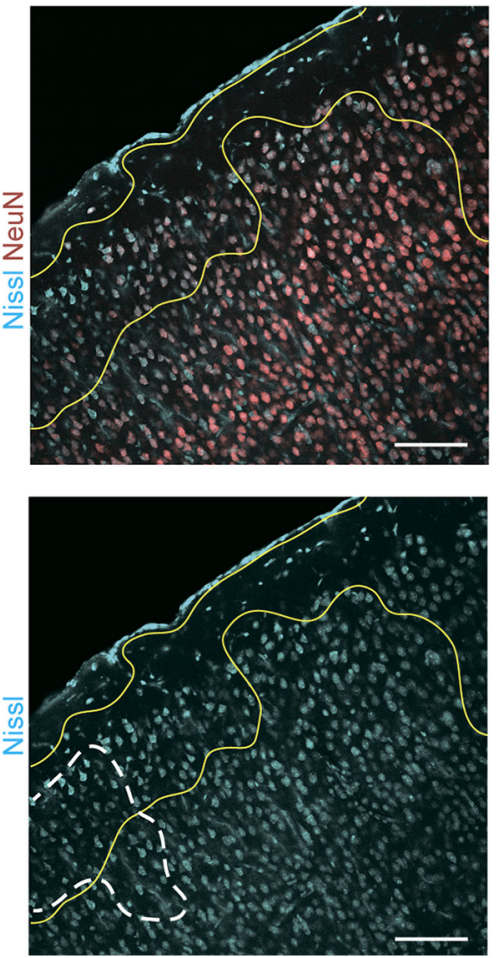

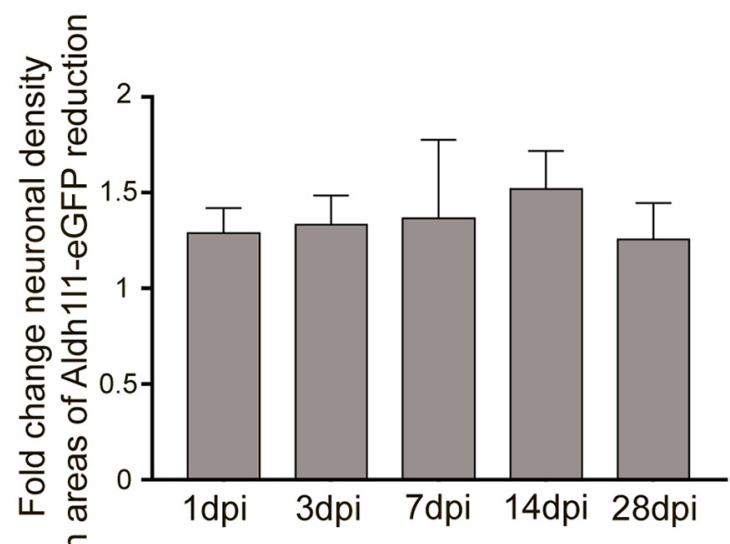

Figure 7. Neuronal densities were unchanged in areas with atypical astrocytes. Areas with atypical astrocytes are indicated by a solid yellow line. $\boldsymbol{a}$, In areas with atypical astrocytes, expression of the pan-neuronal marker NeuN was abnormal. Three distinct patterns were seen (rightmost image): those that were consistent in NeuN intensity outside and appeared normal (1), those that had an overall decrease in NeuN expression (2), and those that formed a ring of NeuN where the center of the soma was void of NeuN (3). $\boldsymbol{b}$, Nissl staining labeled all neurons in areas with abnormal astrocytes at various time points. c, Quantification of neuronal numbers within cortical layers II/III normalized to area size within areas of atypical astrocytes and in peripheral areas. Neuronal densities in immediately adjacent "peripheral" areas were set to 1 and the fold change of densities within areas of loss was computed for each ROI. See Figure 7-1 (available as Extended Data) for breakdown of sample sizes dependent on sex for representative images and detailed numerical data for all statistical group comparisons.

(Fig. 8e) and the distinction was made based on behavioral observations in synchronized video recordings.

Seizure frequencies were calculated including both convulsive and nonconvulsive events starting at the day of the seizure onset until the end of the recording period for each animal and averaged 3 seizures in $10 \mathrm{~d}$. There was notable variability in seizure frequencies from animal to animal (Fig. $8 f$, seizure frequencies compared between $\mathrm{PTE}^{+}, \mathrm{PTE}^{-}$, and Sham mice: one-way ANOVA, $p=0.0042$; Sidak's multiple-comparisons test, $p=0.0073$; Fig. 8-1, available as Extended Data). The earliest seizure was observed at $21 \mathrm{dpi}$. The average latency to the first spontaneous seizure was $38 \pm 7.642 \mathrm{~d}$ (mean \pm SEM).
Areas with atypical astrocytes are larger in animals with PTE Overall no tissue loss was observed in mice with seizures compared with sham mice, but one rdTBI mouse with convulsive seizures had hippocampal atrophy (Fig. 8g; Fig. 8-1, available as Extended Data).

To determine whether there were differences in the astrocyte response between rdTBI animals that incurred seizures and those that did not develop seizures, we quantitatively assessed the size of areas with Glt1 loss as a surrogate for atypical astrocytes. Mice that developed spontaneous seizures (convulsive and nonconvulsive combined) had significantly greater Glt1 area loss fractions compared with mice that underwent rdTBI but did not develop 
a

\section{Seizure Incidence}

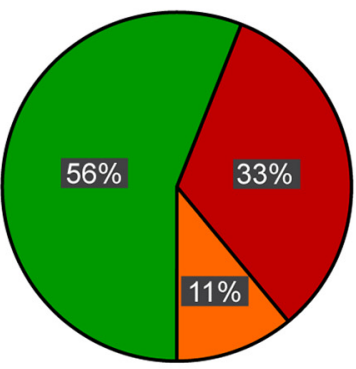

rdTBI, no seizures

raTBI, convulsive seizures rdTBI, non-convulsive seizures

\section{b $3 x$ Sham}

\section{R. Parietal}

L. Parietal

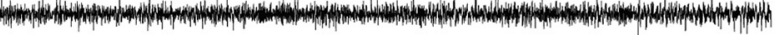

C

rdTBI Convulsive seizure

R. Parietal

L. Parietal

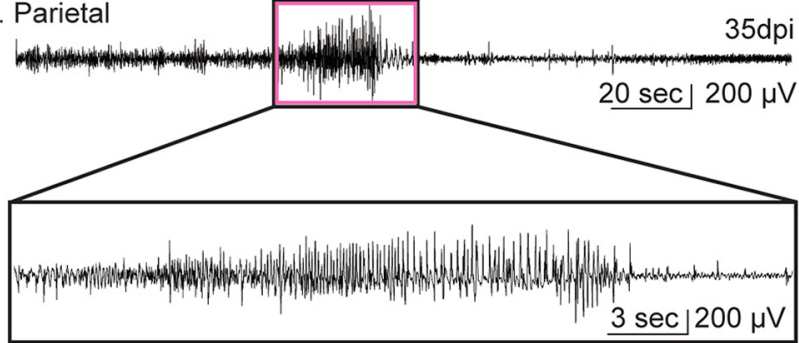

d

rdTBI Non-convulsive seizure

R. Frontal

L. Parietal

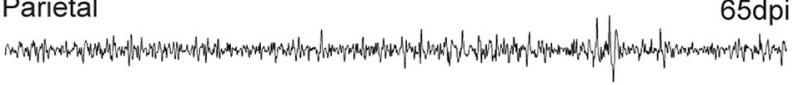

L. Parietal

R. Parietal

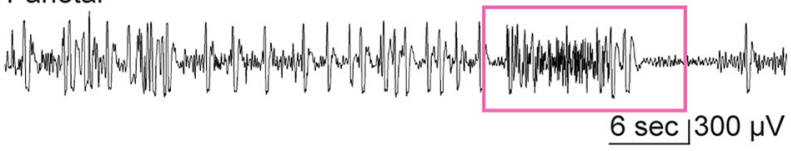

e

$3 \times$ Sham

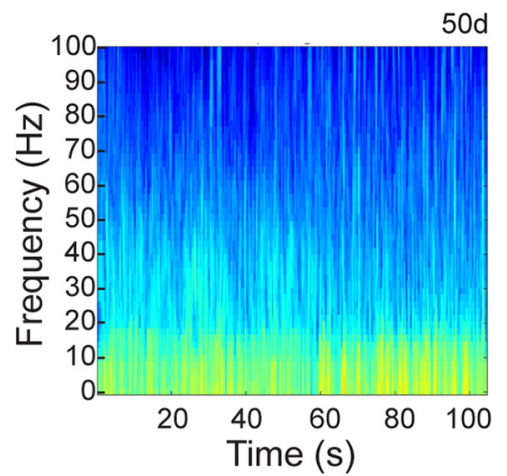

$20 \sec 200 \mu \mathrm{V}$

h

rdTBI, convulsive seizures, large diffuse area
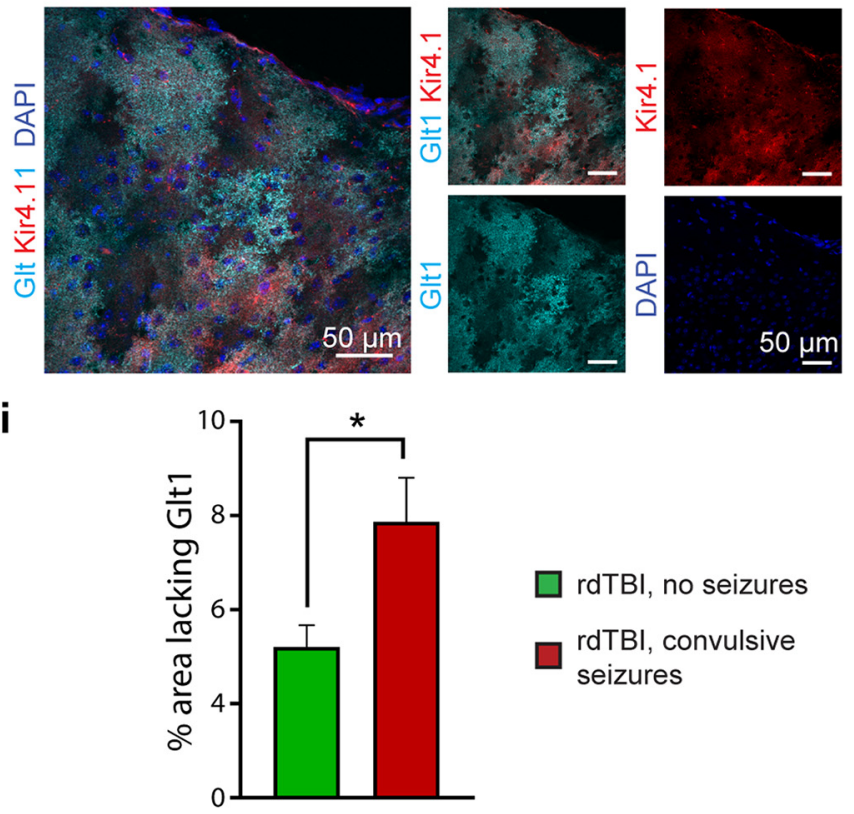

rdTBI, no seizures

rdTBI, convulsive seizures

Figure 8. rdTBI caused spontaneous, unprovoked, recurrent seizures. $\boldsymbol{a}$, Pie chart representing the incidence of seizures in mice with rdTBI. Four of 9 (44\%) mice developed recurrent spontaneous seizures; 3 (33\%) mice developed convulsive seizures; 1 (11\%) mouse developed recurrent spontaneous nonconvulsive seizures; and 5 of $9(56 \%)$ mice had no seizures. $\boldsymbol{b}$, EEG trace of a 3xSham mouse $50 \mathrm{~d}$ after the electrode placement surgery. Channel (Ch) reading: right parietal electrode to left parietal electrode. $c$, EEG trace of a mouse with a spontaneous convulsive seizure $35 \mathrm{dpi}$. The enlarged panel (with time scale 3 s) indicates emergence of high-amplitude evolving repetitive spikes and spike-waves followed by postictal depression (right part of the enlarged panel). Channel reading as in $\boldsymbol{b}$ and $\boldsymbol{d}$, EEG traces of a nonconvulsive seizure at 65 dpi consisting of repetitive high-amplitude spike-and-slow wave discharges in the absence of any (Figure legend continues.) 
seizures (Fig. $8 h, i ; p=0.0432$, unpaired $t$ test; Fig. 8-1, available as Extended Data).

In conclusion, we showed for the first time that repeated diffuse closed-head TBI is sufficient to induce PTE characterized by recurrent spontaneous seizures in a subset of injured mice and that this subset of mice presents with larger areas of atypical astrocytes, suggesting that the rapid changes that astrocytes undergo after rdTBI might be causally linked to epileptogenesis.

\section{Discussion}

Impact acceleration model recapitulates diffuse TBI

Although no animal model fully recapitulates the complex and heterogeneous biomechanics of human TBI, the impact acceleration model used in this study induces diffuse injury, is scalable from mild to severe, and mimics many clinical aspects of closed-head TBI, including diffuse axonal injury and LOC (Foda and Marmarou, 1994; Marmarou et al., 1994, 2009). If calibrated to mild/concussive injury (i.e., in the absence of focal lesions and pronounced neuronal death), sleep disturbances, increased anxiety, and cognitive deficits result (Nichols et al., 2016) and are similar to symptoms reported by patients who incurred a clinically mild/concussive TBI. In our study, single and repetitive impacts of a $100 \mathrm{~g}$ weight from 50 $\mathrm{cm}$ height resulted in prolonged LOC indicative of TBI. However, obvious tissue lesions, hemorrhages, pronounced neuroinflammation, and neuronal death were not caused within the cortical gray matter or hippocampus of mice that were injured once or repetitively. This model thus adequately mimics diffuse injury in the absence of focal TBI.

\section{Heterogeneity of the astrocyte response to brain injury}

A major challenge in characterizing reactive astrocytes is the underappreciated heterogeneity of these cells in the context of brain injury and neurological disease (Zhang and Barres, 2010; Anderson et al., 2014; Robel, 2017). Due to the reliability of the astrogliosis response, it is often thought of as an "all or none" process based solely on the upregulation of GFAP. However, the extent and exact composition of cellular, molecular, and physiological adjustments are not equal for each astrocyte. In fact, different reactive astrocyte types may be protective or neurotoxic (Zamanian et al., 2012; Liddelow et al., 2017). Some reactive astrocytes have been reported to reexpress factors important for synaptogenesis (Zamanian et al., 2012) and axonal guidance (Anderson et al., 2016), possibly aiding in CNS plasticity and repair. Others are characterized by loss of homeostatic functions affecting neuronal health and function negatively (Seifert et al., 2010; Robel and Sontheimer, 2016). Astrogliosis is a plethora of graded alterations to gene and protein expression, the secretome, and morphology (Sofroniew, 2014).

\section{$\leftarrow$}

(Figure legend continued.) movement of the mouse. Channel reading: Ch1 right frontal to left parietal electrode; Ch2 left parietal to right parietal electrode. $\boldsymbol{e}$, EEG power spectrum analysis of normal EEGs and convulsive and nonconvulsive seizures. The power density spectra are color-coded on a logarithmic scale (dB). Pink panels in c-e show the location of the ictal event in the EEG and in the corresponding power spectrum. $f$, Seizure frequency in rdTBI mice with convulsive and nonconvulsive seizures compared with 3xSham. $\boldsymbol{g}$, Histological confirmation of the absence of gross tissue loss induced by either rdTBI or electrode placement surgery. $\boldsymbol{h}$, Diffuse loss of Glt1 and Kir4.1 expression in astrocytes in mice with spontaneous recurrent seizures and quantification of Glt 1 loss in $\boldsymbol{i}$. See Figure 8-1 (available as Extended Data) for breakdown of sample sizes dependent on sex for representative images and detailed numerical data for all statistical group comparisons. ${ }^{*} p \leq 0.05,{ }^{* *} p \leq 0.01$.
Therefore, variable changes modify astrocyte-neuron interactions and modulate neuronal function in highly specific ways that have yet to be fully elucidated. This underscores the necessity to evaluate the astrogliosis response specifically for diffuse TBI. In the absence of a focal injury, our study showed little classic astrogliosis with rather mild upregulation of intermediate filament proteins and the absence of astrocyte proliferation. GFAP ${ }^{+}$, lightly hypertrophic astrocytes were often organized in small groups scattered across the cortical gray matter, yet the location and number of these groups varied from animal to animal. Proteins important for astrocyte homeostatic function appeared unchanged within these areas.

After focal TBI, a gradient of reactive astrocytes develops around the injury site with severely altered reactive astrocytes closest to the site of injury and mildly activated ones at greater distances away from this site. After diffuse TBI, such a gradient was not observed and, instead, we found a different type of reactive astrocyte if astrocyte "reactivity" is defined as the response of astrocytes to brain pathology.

\section{New subtype of reactive astrocytes lacking GFAP expression} Locally distinct from the groups of mildly reactive $\mathrm{GFAP}^{+}$astrocytes, another subgroup of astrocytes rapidly shut down expression of astrocytic proteins, including the glutamate transporter Glt1 and the potassium channel Kir4.1. The astrocyte reporter Aldh111-eGFP responded similarly. This occurred as early as 30 min after a single diffuse TBI, suggesting a time line much faster than classic astrogliosis during which GFAP upregulation and morphological changes happen over the course of several days (Robel et al., 2011b) rather than minutes and was sustained for at least 2 months after injury. We suggest categorizing these astrocytes as a distinct reactive astrocyte subtype based on the obvious changes to their proteome and likely impairment in homeostatic functions, albeit with a notable lack of GFAP upregulation. In fact, the lack of GFAP expression in these cells implicates that GFAP is perhaps not well suited as an all-encompassing marker for reactive astrocytes.

\section{Consequences of atypical reactive astrocytes}

Areas of atypical astrocytes accounted for $\sim 6 \%$ of the cortical gray matter. Based on an average number of 16,000 astrocytes/ $\mathrm{mm}^{3}$ in the mouse cortex (Grosche et al., 2013), this accounts for 960 astrocytes $/ \mathrm{mm}^{3}$. Astrocytes tile the brain with little or no overlap between neighboring cells and each astrocyte covers three or four neuronal soma, hundreds of dendrites, and $\sim 100,000$ synapses (Freeman, 2010). Therefore, a lack of potassium and glutamate transporters within individual astrocyte domains is likely to affect, not only the neuronal soma located within the domain, but also all synaptic connections in these areas.

Numerous studies have provided compelling evidence that classical reactive astrocytes are dysfunctional in their ability to maintain a local ion and transmitter milieu suitable to sustain normal neuronal activity (Olsen and Sontheimer, 2008; Ortinski et al., 2010; Seifert et al., 2010; Robel and Sontheimer, 2016; Robel et al., 2015). These studies make a cogent case for astrocytes being more than just bystanders of the disease and support the hypothesis that changes in astrocyte biology may actively contribute to epilepsy. However, studies pointing to a causal relationship between reactive astrocytes and epilepsy were conducted in animal models that presented with widespread astrogliosis composed of hypertrophic, non-scar-forming, reactive astrocytes (Ortinski et al., 2010; Robel et al., 2015). Whether locally confined and TBI- 
specific changes in astrocytes are sufficient to initiate and drive progression toward PTE remains an unresolved issue. We propose that the early and sustained loss of homeostatic proteins in atypical reactive astrocytes in the cortical gray matter and hippocampus might initiate epileptogenesis and formation of seizure foci. In fact, our data indicate uncoupling of atypical astrocytes and their Glt ${ }^{+}$neighbors possibly due to the lack of $\mathrm{Cx} 43$ expression. Coupling between astrocytes has been implicated in potassium clearance through spatial buffering (Wallraff et al., 2006; Olsen and Sontheimer, 2008; Steinhäuser et al., 2012). However, despite several studies in the field, the role of $\mathrm{Cx} 43$ in epileptogenesis remains controversial (Seifert et al., 2010). Loss of astrocyte coupling is associated with epileptic tissues in humans and animal models of epilepsy (Steinhäuser et al., 2012; Pannasch and Rouach, 2013; Bedner et al., 2015), yet pharmacological studies blocking $\mathrm{Cx} 43$ function suggest that gap junctions may perpetuate epileptiform activity (Samoilova et al., 2008). These opposing results can be explained by the fact that human epilepsy is not a uniform condition, which highlights the importance of proper models used to study such a disorder. Therefore, the specific mechanism by which astrocyte uncoupling might contribute to epileptiform activity after diffuse TBI remains to be elucidated.

\section{Diffuse mild/concussive closed-head TBI in mice is sufficient to induce PTE and seizure development correlates with the presence of atypical astrocytes}

Until now, the field was limited to only two animal models of PTE with confirmed spontaneous seizures (Ostergard et al., 2016). The existing animal models, which induce TBI either by controlled cortical impact or by fluid percussion injury, recapitulate characteristics of focal human TBI and cause PTE in a subgroup of rodents. In these models, however, mortality rates are high, amounting to approximately one-third of the animals (Kharatishvili et al., 2006). Focal injury introduces significant complexity by generating several reactive astrocyte types and initiating other mechanisms involved in seizure generation, such as hemorrhages, tissue necrosis, and large structural lesions in the cerebral cortex (Zhao et al., 2007, 2012; Neshige et al., 2015; Glushakov et al., 2016). There is no doubt that focal injury potentiates the risk for the development of PTE (Annegers and Coan, 2000), likely by all of these mechanisms acting in parallel, and the larger likelihood of immediate and early seizures, which in turn can cause or intensify astrogliosis. Here, we show that repetitive diffuse TBI is sufficient to induce the development of spontaneous seizures 3-4 weeks after TBI even in the absence of hemorrhages, tissue necrosis, and large structural lesions.

Lack of glutamate homeostasis and the resulting increase in excitation or decrease in inhibition has been suggested for other types of epilepsy and epileptic encephalopathies (Ye and Sontheimer, 1998; Ortinski et al., 2010; Buckingham et al., 2011; Robert et al., 2015; Shandra et al., 2017). Our data link for the first time PTE after diffuse TBI with the early and sustained loss of Glt1 expression in associated atypical astrocytes. These areas were significantly larger in animals with seizures compared with seizure-free TBI animals, providing evidence in support of the idea that loss of homeostatic functions in astrocytes may contribute to PTE. Although the exact interplay between astrocytes and neuronal dysfunction leading to epilepsy remain to be clarified, this study provides both the medical and research community with a new model to study the effects of diffuse TBI in initiating epileptogenesis and identifies a cellular mechanism likely to be involved in this process.

\section{References}

Abou-Abbass H, Bahmad H, Ghandour H, Fares J, Wazzi-Mkahal R, Yacoub B, Darwish H, Mondello S, Harati H, El Sayed MJ, Tamim H, Kobeissy F (2016) Epidemiology and clinical characteristics of traumatic brain injury in Lebanon: a systematic review. Medicine 95:e5342.

Anderson MA, Ao Y, Sofroniew MV (2014) Heterogeneity of reactive astrocytes. Neurosci Lett 565:23-29.

Anderson MA, Burda JE, Ren Y, Ao Y, O'Shea TM, Kawaguchi R, Coppola G, Khakh BS, Deming TJ, Sofroniew MV (2016) Astrocyte scar formation aids central nervous system axon regeneration. Nature 532:195-200.

Annegers JF, Coan SP (2000) The risks of epilepsy after traumatic brain injury. Seizure 9:453-457.

Annegers JF, Grabow JD, Groover RV, Laws ER Jr, Elveback LR, Kurland LT (1980) Seizures after head trauma: a population study. Neurology 30:683-689.

Annegers JF, Hauser WA, Coan SP, Rocca WA (1998) A population-based study of seizures after traumatic brain injuries. N Engl J Med 338:20-24.

Bedner P, Dupper A, Hüttmann K, Müller J, Herde MK, Dublin P, Deshpande T, Schramm J, Häussler U, Haas CA, Henneberger C, Theis M, Steinhäuser C (2015) Astrocyte uncoupling as a cause of human temporal lobe epilepsy. Brain 138:1208-1222.

Bouchat J, Couturier B, Marneffe C, Gankam-Kengne F, Balau B, De Swert K, Brion JP, Poncelet L, Gilloteaux J, Nicaise C (2018) Regional oligodendrocytopathy and astrocytopathy precede myelin loss and blood-brain barrier disruption in a murine model of osmotic demyelination syndrome. Glia 66:606-622.

Buckingham SC, Campbell SL, Haas BR, Montana V, Robel S, Ogunrinu T, Sontheimer H (2011) Glutamate release by primary brain tumors induces epileptic activity. Nat Med 17:1269-1274.

Buffo A, Rite I, Tripathi P, Lepier A, Colak D, Horn AP, Mori T, Götz M (2008) Origin and progeny of reactive gliosis: a source of multipotent cells in the injured brain. Proc Natl Acad Sci U S A 105:3581-3586.

Bush TG, Puvanachandra N, Horner CH, Polito A, Ostenfeld T, Svendsen CN, Mucke L, Johnson MH, Sofroniew MV (1999) Leukocyte infiltration, neuronal degeneration, and neurite outgrowth after ablation of scar-forming, reactive astrocytes in adult transgenic mice. Neuron 23:297-308.

Christensen J (2012) Traumatic brain injury: risks of epilepsy and implications for medicolegal assessment. Epilepsia 53:43-47.

Christensen J, Pedersen MG, Pedersen CB, Sidenius P, Olsen J, Vestergaard M (2009) Long-term risk of epilepsy after traumatic brain injury in children and young adults: a population-based cohort study. Lancet 373:11051110.

Condamine S, Verdier D, Kolta A (2018) Analyzing the size, shape, and directionality of networks of coupled astrocytes. J Vis Exp 140.

Dermietzel R, Hertberg EL, Kessler JA, Spray DC (1991) Gap junctions between cultured astrocytes: immunocytochemical, molecular, and electrophysiological analysis. J Neurosci 11:1421-1432.

Faulkner JR, Herrmann JE, Woo MJ, Tansey KE, Doan NB, Sofroniew MV (2004) Reactive astrocytes protect tissue and preserve function after spinal cord injury. J Neurosci 24:2143-2155.

Ferguson PL, Smith GM, Wannamaker BB, Thurman DJ, Pickelsimer EE, Selassie AW (2010) A population-based study of risk of epilepsy after hospitalization for traumatic brain injury. Epilepsia 51:891-898.

Foda MA, Marmarou A (1994) A new model of diffuse brain injury in rats. part II: morphological characterization. J Neurosurg 80:301-313.

Foresti ML, Arisi GM, Shapiro LA (2011) Role of glia in epilepsy-associated neuropathology, neuroinflammation and neurogenesis. Brain Res Rev $66: 115-122$

Freeman MR (2010) Specification and morphogenesis of astrocytes. Science 330:774-778

Frey LC (2003) Epidemiology of posttraumatic epilepsy: a critical review. Epilepsia 44:11-17.

Gee JM, Smith NA, Fernandez FR, Economo MN, Brunert D, Rothermel M, Morris SC, Talbot A, Palumbos S, Ichida JM, Shepherd JD, West PJ, Wachowiak M, Capecchi MR, Wilcox KS, White JA, Tvrdik P (2014) Imaging activity in neurons and glia with a Polr2a-based and credependent GCaMP5G-IRES-tdTomato reporter mouse. Neuron 83: $1058-1072$

Glushakov AV, Glushakova OY, Doré S, Carney PR, Hayes RL (2016) Animal models of posttraumatic seizures and epilepsy. Methods Mol Biol 1462:481-519. 
Götz M, Sirko S, Beckers J, Irmler M (2015) Reactive astrocytes as neural stem or progenitor cells: in vivo lineage, in vitro potential, and genomewide expression analysis. Glia 63:1452-1468.

Griemsmann S, Höft SP, Bedner P, Zhang J, von Staden E, Beinhauer A, Degen J, Dublin P, Cope DW, Richter N, Crunelli V, Jabs R, Willecke K, Theis M, Seifert G, Kettenmann H, Steinhäuser C (2015) Characterization of panglial gap junction networks in the thalamus, neocortex, and hippocampus reveals a unique population of glial cells. Cereb Cortex 25:3420-3433.

Grosche A, Grosche J, Tackenberg M, Scheller D, Gerstner G, Gumprecht A, Pannicke T, Hirrlinger PG, Wilhelmsson U, Hüttmann K, Härtig W, Steinhäuser C, Pekny M, Reichenbach A (2013) Versatile and simple approach to determine astrocyte territories in mouse neocortex and hippocampus. PLoS One 8:e69143.

Gusel'nikova VV, Korzhevskiy DE (2015) NeuN as a neuronal nuclear antigen and neuron differentiation marker. Acta Naturae 7:42-47.

Herrmann JE, Imura T, Song B, Qi J, Ao Y, Nguyen TK, Korsak RA, Takeda K, Akira S, Sofroniew MV (2008) STAT3 is a critical regulator of astrogliosis and scar formation after spinal cord injury. J Neurosci 28:7231-7243.

Jany PL, Hagemann TL, Messing A (2013) GFAP expression as an indicator of disease severity in mouse models of alexander disease. ASN Neuro 5:e00109.

Kharatishvili I, Nissinen JP, McIntosh TK, Pitkänen A (2006) A model of posttraumatic epilepsy induced by lateral fluid-percussion brain injury in rats. Neuroscience 140:685-697.

Komar AA, Hatzoglou M (2011) Cellular IRES-mediated translation: the war of ITAFs in pathophysiological states. Cell Cycle 10:229-240.

Konietzko U, Müller CM (1994) Astrocytic dye coupling in rat hippocampus: topography, developmental onset, and modulation by protein kinase C. Hippocampus 4:297-306.

Kumar R, Gupta RK, Husain M, Vatsal DK, Chawla S, Rathore RK, Pradhan S (2003) Magnetization transfer MR imaging in patients with posttraumatic epilepsy. AJNR Am J Neuroradiol 24:218-224.

Levine J, Kwon E, Paez P, Yan W, Czerwieniec G, Loo JA, Sofroniew MV, Wanner IB (2016) Traumatically injured astrocytes release a proteomic signature modulated by STAT3-dependent cell survival. Glia 64:668694.

Liddelow SA, Guttenplan KA, Clarke LE, Bennett FC, Bohlen CJ, Schirmer L, Bennett ML, Münch AE, Chung WS, Peterson TC, Wilton DK, Frouin A, Napier BA, Panicker N, Kumar M, Buckwalter MS, Rowitch DH, Dawson VL, Dawson TM1, Stevens B et al. (2017) Neurotoxic reactive astrocytes are induced by activated microglia. Nature 541:481-487.

Lioy DT, Garg SK, Monaghan CE, Raber J, Foust KD, Kaspar BK, Hirrlinger PG, Kirchhoff F, Bissonnette JM, Ballas N, Mandel G (2011) A role for glia in the progression of Rett's syndrome. Nature 475:497-500.

Lowenstein DH (2009) Epilepsy after head injury: an overview. Epilepsia $50: 4-9$.

Mader EC Jr, Villemarette-Pittman NR, Kashirny SV, Santana-Gould L, Olejniczak PW (2012) Typical spike-and-wave activity in hypoxic-ischemic brain injury and its implications for classifying nonconvulsive status epilepticus. Clin Med Insights Case Rep 5:99-106.

Management of Concussion/mTBI Working Group (2009) VA/DoD clinical practice guideline for management of concussion/mild traumatic brain injury. J Rehabil Res Dev 46:CP1-CP68.

Marks DA, Kim J, Spencer DD, Spencer SS (1995) Seizure localization and pathology following head injury in patients with uncontrolled epilepsy. Neurology 45:2051-2057.

Marmarou A, Foda MA, van den Brink W, Campbell J, Kita H, Demetriadou K (1994) A new model of diffuse brain injury in rats. part I: pathophysiology and biomechanics. J Neurosurg 80:291-300.

Marmarou CR, Prieto R, Taya K, Young HF, Marmarou A (2009) Marmarou weight drop injury model. In: Animal models of acute neurological injuries, pp 393-407. Totowa, NJ: Humana.

Morehead M, Bartus RT, Dean RL, Miotke JA, Murphy S, Sall J, Goldman H (1994) Histopathologic consequences of moderate concussion in an animal model: correlations with duration of unconsciousness. J Neurotrauma 11:657-667.

National Center for Injury Prevention and Control-Centers for Disease Control and Prevention (2003) Report to congress on mild traumatic brain injury in the United States: steps to prevent a serious public health prob- lem. Available from: https://www.cdc.gov/traumaticbraininjury/pdf/ mtbireport-a.pdf.

Neshige S, Kuriyama M, Yoshimoto T, Takeshima S, Himeno T, Takamatsu K, Sato M, Ota S (2015) Seizures after intracerebral hemorrhage; risk factor, recurrence, efficacy of antiepileptic drug. J Neurol Sci 359:318-322.

Nichols JN, Deshane AS, Niedzielko TL, Smith CD, Floyd CL (2016) Greater neurobehavioral deficits occur in adult mice after repeated, as compared to single, mild traumatic brain injury (mTBI). Behav Brain Res 298:111-124.

Niquet J, Ben-Ari Y, Represa A (1994) Glial reaction after seizure induced hippocampal lesion: immunohistochemical characterization of proliferating glial cells. J Neurocytol 23:641-656.

Olsen ML, Sontheimer H (2008) Functional implications for Kir4.1 channels in glial biology: from $\mathrm{K}^{+}$buffering to cell differentiation. J Neurochem 107:589-601.

Ortinski PI, Dong J, Mungenast A, Yue C, Takano H, Watson DJ, Haydon PG, Coulter DA (2010) Selective induction of astrocytic gliosis generates deficits in neuronal inhibition. Nat Neurosci 13:584-591.

Ostergard T, Sweet J, Kusyk D, Herring E, Miller J (2016) Animal models of post-traumatic epilepsy. J Neurosci Methods 272:50-55.

Pannasch U, Rouach N (2013) Emerging role for astroglial networks in information processing: from synapse to behavior. Trends Neurosci 36:405-417.

Pekny M, Pekna M, Messing A, Steinhäuser C, Lee JM, Parpura V, Hol EM, Sofroniew MV, Verkhratsky A (2016) Astrocytes: a central element in neurological diseases. Acta Neuropathol 131:323-345.

Pitkänen A, Immonen R (2014) Epilepsy related to traumatic brain injury. Neurotherapeutics 11:286-296.

Pollen DA, Trachtenberg MC (1970) Neuroglia: gliosis and focal epilepsy. Science 167:1252-1253.

Robel S (2017) Astroglial scarring and seizures: a cell biological perspective on epilepsy. Neuroscientist 23:152-168.

Robel S, Sontheimer H (2016) Glia as drivers of abnormal neuronal activity. Nat Neurosci 19:28-33.

Robel S, Bardehle S, Lepier A, Brakebusch C, Götz M (2011a) Genetic deletion of cdc 42 reveals a crucial role for astrocyte recruitment to the injury site in vitro and in vivo. J Neurosci 31:12471-12482.

Robel S, Berninger B, Götz M (2011b) The stem cell potential of glia: lessons from reactive gliosis. Nat Rev Neurosci 12:88-104.

Robel S, Buckingham SC, Boni JL, Campbell SL, Danbolt NC, Riedemann T, Sutor B, Sontheimer H (2015) Reactive astrogliosis causes the development of spontaneous seizures. J Neurosci 35:3330-3345.

Robert SM, Buckingham SC, Campbell SL, Robel S, Holt KT, OgunrinuBabarinde T, Warren PP, White DM, Reid MA, Eschbacher JM, Berens ME, Lahti AC, Nabors LB, Sontheimer H (2015) SLC7A11 expression is associated with seizures and predicts poor survival in patients with malignant glioma. Sci Transl Med 7:289ra86.

Samoilova M, Wentlandt K, Adamchik Y, Velumian AA, Carlen PL (2008) Connexin 43 mimetic peptides inhibit spontaneous epileptiform activity in organotypic hippocampal slice cultures. Exp Neurol 210:762-775.

Seifert G, Carmignoto G, Steinhäuser C (2010) Astrocyte dysfunction in epilepsy. Brain Res Rev 63:212-221.

Shandra O, Robel S (2019) Imaging and manipulating astrocyte function in vivo in the context of CNS injury. Methods Mol Biol 2019 1938:233-246.

Shandra O, Moshé SL, Galanopoulou AS (2017) Inflammation in epileptic encephalopathies. Adv Protein Chem Struct Biol 108:59-84.

Simon C, Götz M, Dimou L (2011) Progenitors in the adult cerebral cortex: cell cycle properties and regulation by physiological stimuli and injury. Glia 59:869-881.

Sofroniew MV (2009) Molecular dissection of reactive astrogliosis and glial scar formation. Trends Neurosci 32:638-647.

Sofroniew MV (2014) Astrogliosis. Cold Spring Harb Perspect Biol 7:a020420.

Sofroniew MV (2015) Astrocyte barriers to neurotoxic inflammation. Nat Rev Neurosci 16:249-263.

Steinhäuser C, Seifert G, Bedner P (2012) Astrocyte dysfunction in temporal lobe epilepsy: $\mathrm{K}^{+}$channels and gap junction coupling. Glia 60:1192-1202.

Tong X, Ao Y, Faas GC, Nwaobi SE, Xu J, Haustein MD, Anderson MA, Mody I, Olsen ML, Sofroniew MV, Khakh BS (2014) Astrocyte Kir4.1 ion channel deficits contribute to neuronal dysfunction in Huntington's disease model mice. Nat Neurosci 17:694-703. 
Verkhratsky A, Sofroniew MV, Messing A, deLanerolle NC, Rempe D, Rodríguez JJ, Nedergaard M (2012) Neurological diseases as primary gliopathies: a reassessment of neurocentrism. ASN Neuro 4:e00082.

Vijayan VK, Lee YL, Eng LF (1990) Increase in glial fibrillary acidic protein following neural trauma. Mol Chem Neuropathol 13:107-118.

Wallraff A, Köhling R, Heinemann U, Theis M, Willecke K, Steinhäuser C (2006) The impact of astrocytic gap junctional coupling on potassium buffering in the hippocampus. J Neurosci 26:5438-5447.

Wennberg R, Hiploylee C, Tai P, Tator CH (2018) Is concussion a risk factor for epilepsy? Can J Neurol Sci 45:275-282.

Wetherington J, Serrano G, Dingledine R (2008) Astrocytes in the epileptic brain. Neuron 58:168-178.

Ye ZC, Sontheimer H (1998) Glial glutamate transport as target for nitric oxide: consequences for neurotoxicity. Prog Brain Res 118:241-251.
Zamanian JL, Xu L, Foo LC, Nouri N, Zhou L, Giffard RG, Barres BA (2012) Genomic analysis of reactive astrogliosis. J Neurosci 32:63916410.

Zhang Y, Barres BA (2010) Astrocyte heterogeneity: an underappreciated topic in neurobiology. Curr Opin Neurobiol 20:588-594.

Zhang Z (2016) Variable selection with stepwise and best subset approaches. Ann Transl Med 4:136.

Zhao M, Suh M, Ma H, Perry C, Geneslaw A, Schwartz TH (2007) Focal increases in perfusion and decreases in hemoglobin oxygenation precede seizure onset in spontaneous human epilepsy. Epilepsia 48:2059-2067.

Zhao Y, Wu H, Wang X, Li J, Zhang S (2012) Clinical epidemiology of posttraumatic epilepsy in a group of chinese patients. Seizure 21:322326. 